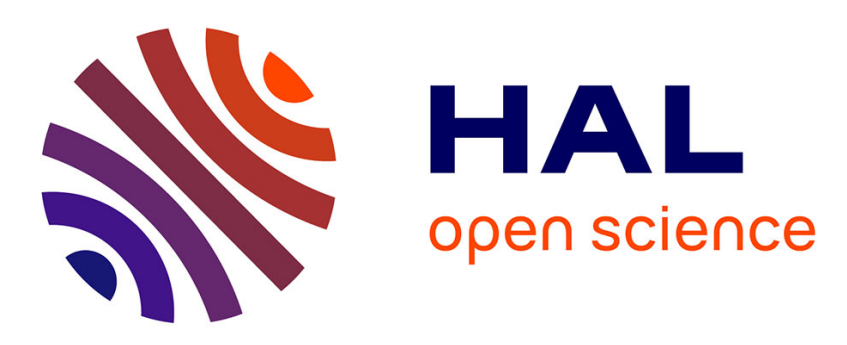

\title{
Empirical Selection of Auroral Kilometric Radiation During a Multipoint Remote Observation With Wind and Cassini
}

\author{
J. Waters, C. Jackman, L. Lamy, B. Cecconi, D. Whiter, X. Bonnin, K. \\ Issautier, A. Fogg
}

\section{To cite this version:}

J. Waters, C. Jackman, L. Lamy, B. Cecconi, D. Whiter, et al.. Empirical Selection of Auroral Kilometric Radiation During a Multipoint Remote Observation With Wind and Cassini. Journal of Geophysical Research Space Physics, 2021, 126 (10), 10.1029/2021JA029425 . hal-03451507

\author{
HAL Id: hal-03451507 \\ https://hal.science/hal-03451507
}

Submitted on 9 Dec 2021

HAL is a multi-disciplinary open access archive for the deposit and dissemination of scientific research documents, whether they are published or not. The documents may come from teaching and research institutions in France or abroad, or from public or private research centers.
L'archive ouverte pluridisciplinaire HAL, est destinée au dépôt et à la diffusion de documents scientifiques de niveau recherche, publiés ou non, émanant des établissements d'enseignement et de recherche français ou étrangers, des laboratoires publics ou privés. 


\section{JGR Space Physics}

\section{RESEARCH ARTICLE}

10.1029/2021JA029425

Key Points:

- Novel, empirically based method to extract Auroral Kilometric Radiation (AKR) from Wind/ WAVES is presented and applied to observations made during the Cassini flyby

- Selected data show a distribution of AKR power with expected longitudinal and latitudinal visibility constraints

- Diurnal temporal modulation observed, suggesting the dominance of a geometric viewing effect and agreeing with previous AKR observations

Correspondence to:

J. E. Waters,

J.Waters@soton.ac.uk

Citation:

Waters, J. E., Jackman, C. M., Lamy, L., Cecconi, B., Whiter, D. K., Bonnin, X., et al. (2021). Empirical selection of Auroral Kilometric Radiation during a multipoint remote observation with wind and Cassini. Journal of Geophysical Research: Space Physics, 126, e2021JA029425. https://doi. org/10.1029/2021JA029425

Received 13 APR 2021 Accepted 28 SEP 2021
(C)2021. The Authors.

This is an open access article under the terms of the Creative Commons Attribution License, which permits use, distribution and reproduction in any medium, provided the original work is properly cited.

\section{Empirical Selection of Auroral Kilometric Radiation During a Multipoint Remote Observation With Wind and Cassini}

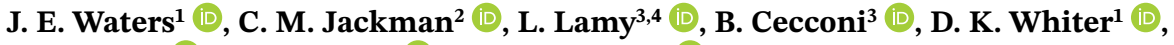 \\ X. Bonnin ${ }^{3}$ (D), K. Issautier ${ }^{3}$ (D), and A. R. Fogg ${ }^{\mathbb{D}}$
}

${ }^{1}$ Space Environment Physics Group, School of Physics and Astronomy, University of Southampton, Southampton, UK, ${ }^{2}$ School of Cosmic Physics, DIAS Dunsink Observatory, Dublin Institute for Advanced Studies, Dublin, Ireland, ${ }^{3}$ LESIA, Observatoire de Paris, PSL Research University, CNRS, Sorbonne Université, Université de Paris, Meudon, France, ${ }^{4}$ LAM, Pythéas, Aix Marseille Université, CNRS, CNES, Marseille, France

Abstract Auroral Kilometric Radiation (AKR) is terrestrial radio emission that originates in particle acceleration regions along magnetic field lines, coinciding with discrete auroral arcs. AKR viewing geometry is complex due to the confinement of the source regions to nightside local times (LTs) and the anisotropy of the beaming pattern, so observations are highly dependent on spacecraft viewing position. We present a novel, empirical technique that selects AKR emission from observations made with the spin-axis aligned antenna of the Wind/WAVES instrument, based on the rapidly varying amplitude of AKR across spacecraft spin timescales. We apply the technique to Wind/WAVES data during 1999 day of year 227-257, when the Cassini spacecraft flew past Earth and provided an opportunity to observe AKR from two remote locations. We examine the AKR flux and power, with observations made from LTs of 1700-0300 hr having an average power up to $10^{4} \mathrm{Wsr}^{-1}$ larger than those on the dayside and an increasing AKR power observed at higher magnetic latitudes. We perform a linear cross-correlation between the Wind AKR power and the spacecraft magnetic latitude, showing positive then negative correlation as Wind travels from the Northern to Southern magnetic hemisphere. Statistically significant diurnal modulations are found in the whole 30-day period and in subsets of the data covering different local time sectors, indicative of a predominantly geometrical effect for remote AKR viewing. The reproduction of well-known features of the AKR verifies the empirical selection and shows the promise of its application to Wind/WAVES observations.

Plain Language Summary Auroral Kilometric Radiation (AKR) is naturally occurring radio emission from the Earth's Northern and Southern polar regions, which becomes more intense as the aurora brightens. In this work, we examine data from the Wind spacecraft WAVES instrument from a 30- day interval in 1999 when a second spacecraft, Cassini, was also flying near Earth and measuring the AKR from a different viewpoint. In this work, we select the AKR using an empirical measure of the variability observed by the WAVES instrument, and compare the distribution and time profile of AKR intensity. Comparing measurements of this radio emission from different spacecraft positions help us to understand how the AKR is best viewed and illustrate the constrained beaming of the emission. This information is important for anyone wanting to attempt to interpret measurements of the AKR.

\section{Introduction}

Auroral kilometric radiation (AKR) describes amplified radio emission from the Earth that is generated from relativistic, precipitating electrons along magnetic field lines in the auroral zone and resonates at the electron cyclotron frequency (Wu \& Lee, 1979). The emission frequency of an AKR source is close to the local electron gyrofrequency, so that lower frequency AKR emanates from a higher altitude along a field line. AKR is emitted between $\sim 30-800 \mathrm{kHz}$ and has been observed by many Earth-orbiting spacecraft such as Polar, Geotail, and Cluster (e.g., Anderson et al., 2005; Liou et al., 2000; Morioka et al., 2007; Mutel et al., 2003). The emission mechanism, the electron cyclotron maser instability, is such that AKR is emitted at angles near-perpendicular to the field lines. This leads to largely anisotropic beaming of AKR from individual field lines that has been constrained both through modeling and observations. Earliest observations 
suggested that AKR can be observed within a cone at angles that are increasingly oblique to the magnetic field with decreasing frequency (Green et al., 1977; Kasaba et al., 1997). However, more recent observations using Cluster suggest that AKR is emitted in a more restricted geometry, with similar longitudinal extent up to a few thousand $\mathrm{km}$ but emitted over a narrower latitudinal region of a few tens of degrees over the auroral zone (Mutel et al., 2008). Both models of the emission geometry have been supported by in-situ observations with the Polar spacecraft, confirming the frequency-dependent, anisotropic beaming of AKR from the source region (Menietti et al., 2011).

Furthermore, AKR is known to be fully circularly polarized, with the handedness depending on the direction of electron gyration in either hemisphere (Kaiser et al., 1978). Where polarization information is available, we expect to see left-handed circularly polarized (LH) emission from the Southern magnetic (Northern geographic) hemisphere and right-handed circularly polarized (RH) emission from the Northern magnetic (Southern geographic) hemisphere (assuming emission in the extraordinary mode). This has been observed at both Earth and Saturn and is a consequence of the emission mechanism (Lamy et al., 2010; Lamy, Zarka, Cecconi, Prangé, et al., 2008). The visibility of AKR is a strong function of the position of the observer. AKR and its source regions are mostly concentrated at nightside local times (LTs); AKR has been observed consistently from LTs between $\sim 1600-0300 \mathrm{hr}$, whereas the most intense source regions are located at 2100-2200 LT (e.g., Alexander \& Kaiser, 1976; Gurnett, 1974; Panchenko, 2003). The visibility of AKR to a spacecraft at various latitudes is constrained by the beaming of the emission, as mentioned above. Ray tracing has been used previously to examine the general propagation of AKR from the source region as well as for instances where the emission may refract from the dense plasmasphere, for example (Green et al., 1977; Mutel et al., 2004). AKR visibility with latitude has also been examined statistically for studies of hemispheric conjugacy using multiple spacecraft (Morioka et al., 2011). For a spacecraft near the equator, it is possible to observe AKR emission from both hemispheres as the emission cones from sources on a given meridian overlap. In this case, the emission from each pole cannot be separated (without polarization information) and the observations must be interpreted as a global average. For closer radial distances, a spacecraft near the equator can be beneath the superposed emission cones of each hemisphere and observe no AKR. At Saturn, this equatorial shadow zone has been modeled and found at radial distances of $<3.6 R_{s}$, where $R_{S}=60268 \mathrm{~km}$ is the radius of Saturn (Lamy, Zarka, Cecconi, Hess, \& Prangé, 2008). At Earth, Morioka et al. (2011) attributed an approximate limit of $7 R_{E}$ to the equatorial shadow zone, where $R_{E}=6371 \mathrm{~km}$ is the radius of Earth.

Given that AKR generation is intrinsic to the magnetic field, the tilt of the planetary field with respect to the rotation axis combines with the highly directive AKR beaming to produce an illumination region that is time-dependent. Temporally, significant periodicities have been found at semi-diurnal and diurnal timescales, the latter of which has been attributed to geometrical viewing effects as the emission region precesses, like the magnetic dipole, with respect to the rotation of the Earth (Lamy et al., 2010). Other suggestions for the source of this modulation include an intrinsic modulation due to the effect on the ionosphere of the tilt of the magnetic dipole with respect to the incoming solar wind (Panchenko et al., 2009) or a physical origin within the magnetosphere itself (Morioka et al., 2013). Discerning the origin of this variability is useful to further the understanding of the magnetosphere-ionosphere coupling.

In this study, we are concerned with the extraction of AKR from the raw data of an Earth-orbiting spacecraft, as well as the interpretation and quantification of any visibility effects due to the location of the spacecraft relative to the radio sources. Moreover, the AKR has the potential to serve as an excellent diagnostic tool both for solar wind driving and for magnetospheric dynamics. Specifically, previous work has shown that AKR can intensify during periods of magnetospheric disturbance (Liou et al., 2000; Voots et al., 1977; Zhao et al., 2019). The generation of AKR requires the presence of strong, parallel electric fields that accelerate electrons to the necessary relativistic speeds within the magnetosphere-ionosphere coupling region. The well-studied physical phenomenon of the magnetospheric substorm manifests in various observable signatures in both the magnetosphere and ionosphere. In the magnetosphere, the magnetic field dipolarizes following reconnection in the magnetotail and energetic plasma flows Earthward (Juusola et al., 2011; Liou, 2002). Energetic particles are injected into the ionosphere as the substorm current wedge strengthens the current systems at high latitudes, brightening the aurora and causing well known morphological changes in the oval (Akasofu, 1964; Kepko et al., 2015; McPherron et al., 1973). For AKR, not only does the 
emission intensify but the frequency spectrum undergoes characteristic changes in response to substorm behavior. Observations with the Polar spacecraft have shown that the AKR source region morphology may have a dual structure, suggesting that a given field line has a more persistent AKR source at lower altitudes that suddenly extends to higher altitudes at the time of substorm onset (Morioka et al., 2007).

Before the properties of AKR can be studied in detail, the AKR-related radio signals must be disentangled from other radio emissions detected by a spacecraft's radio instrument. This non-trivial process is described in more detail in Section 2 below. Broadly speaking, an orbiting spacecraft may detect multiple possible sources of radio emission at multiple wavelengths when surveying the radio environment. At kilometric wavelengths, corresponding to frequencies of $\sim \mathrm{MHz}$ and below, the long, drifting tails of solar radio type III bursts can be observed, which are ubiquitous when the spacecraft is in the solar wind (Krupar et al., 2018). As well as this, characteristic frequencies of the local plasma can be observed at lower frequencies. This can occur both in the solar wind, at the plasma frequency and harmonics following Langmuir waves, or within the magnetosphere, where dense, turbulent plasma in the magnetosheath leads to a rise in quasi-thermal noise (QTN) (Meyer-Vernet et al., 1998, 2017). Thus AKR is often observed in superposition with other waves and must be explicitly selected, where possible, for a complete study. Goniopolarimetric (GP) inversion techniques are useful for selecting AKR, as the Stokes parameters of an incident wave can be derived using a model that accounts for the geometry of both the radio antennae and the source (Cecconi, 2019; Cecconi \& Zarka, 2005). Then, the circular polarization can be used to discriminate against other sources; the few observations of solar radio Type III bursts at frequencies $<1 \mathrm{MHz}$ show weak polarization (Pulupa et al., 2019; Reiner et al., 2007). This has been done at Saturn, using the radio instrument on board the three-axis stabilized Cassini spacecraft to observe SKR, the Kronian analog of AKR. As the Cassini spacecraft flew by Earth, its radio instrument was turned on for a month-long period. During this time, the instrument was used to retrieve the circular polarization state of the AKR, allowing the general emission characteristics, such as the emission power and the temporal modulation to be studied (Lamy et al., 2010). For this month-long period, the Wind spacecraft was traveling on orbits that carried it through the nightside magnetosphere at perigee, allowing it to make remote observations of the AKR source region, as well as other opportunities to observe AKR from other LTs. Although it is not possible to apply previously developed GP techniques for spinning spacecraft to AKR observations with Wind, a selection technique based on the observed variability on timescales of seconds has been developed and applied. This has provided an effective selection of AKR emission, allowing a quantitative analysis and comparison to be performed. Here, we focus on the unique dual vantage point of this Cassini-Wind conjunction during 1999. In Section 2, we describe the instrumentation, the calibration of the radio data, and the selection technique, which we have applied to extract AKR. In Section 3, we compare and contrast the viewing geometry and observations of Wind and Cassini as they traverse the terrestrial magnetosphere on different paths. In Section 4, we summarize our findings and interpretation of the complementary data.

\section{Instrumentation and Empirical Data Selection Technique}

The Wind spacecraft, launched in 1994 as part of the International Solar Terrestrial Physics (ISTP) mission, is equipped with various instruments designed to study the solar wind and radio emissions from both the Sun and Earth. The primary function of the spacecraft is that of a solar wind monitor, and Wind has most often observed from the Lagrangian point L1 (sunward of Earth); Wind first reached L1 in 1996 before spending time between 1998 and 2004 executing complex orbital maneuvers to explore Earth's magnetosphere. The spacecraft returned to L1 in 2004 and has been there since. The relevant instrumentation will be described first in Section 2.1 before the appropriate calibration steps are described in Section 2.2. The method of selecting radio data pertaining to AKR is then illustrated in Section 2.3.

\subsection{Wind/WAVES Radio Instrumentation}

The WAVES investigation (Bougeret et al., 1995) is comprised of two antennae in the spin plane of the spacecraft ( $\mathrm{X}$ and $\mathrm{Y}$ - with original tip-to-tip lengths of 100 and $15 \mathrm{~m}$, respectively) as well as one aligned with the spin axis ( $\mathrm{Z}$, of length $12 \mathrm{~m}$ tip-to-tip). The antennae used here are of the electric dipole type, each formed of two monopolar wire lengths along the same axis on either side of the spacecraft, with the $Z$ antenna having a rigid structure. Of the three, WAVES radio receivers RAD1 operates between 20 and $1,040 \mathrm{kHz}$ 
covering the whole AKR frequency spectrum and utilizes antennae that are each in the short-antenna regime, allowing for a beaming pattern that is independent of the observation frequency.

The RAD1 receiver can operate in one of two modes: the SEP mode allows the receiver to measure with one of the equatorial antennae (usually $\mathrm{X}$ ) and the $\mathrm{Z}$ antenna independently. The SUM mode performs the electronic summation of the $\mathrm{X}$ and $\mathrm{Z}$ antennae, outputting this synthetic signal as well as one with a $\frac{\pi}{2}$ phase shift applied to the equatorial antenna. The SUM mode thus returns two signals from the synthetic inclined dipole. In this work, the original SUM signal will be referred to as the S antenna and that with a phase shift applied as the S' antenna. RAD1 has 256 available frequency channels between 20 and 1,040 kHz and channels can be chosen to sample the radio environment using three different methods. The most often used allows the instrument to be provided with a list of frequencies to be measured over the next fixed-duration sweep cycle of samples.

Each frequency channel is measured at each antenna over the respective integration time (154 ms for the $\mathrm{S}$ and $\mathrm{S}$ ' antennae and $308 \mathrm{~ms}$ for the $\mathrm{Z}$ antenna) to comprise a single observation. Measurements are then repeated at that frequency across a spacecraft spin period of $3 \mathrm{~s}$ in order to receive a signal that corresponds to a single period of modulation. Sampling all 256 frequency channels, while offering greater spectral resolution, would increase the duration of the sweep cycle and so decrease the temporal resolution. The typical total time attributed to the measurement of a single frequency for S, S', and Z antennae, accounting for the offset incurred at the beginning of the frequency sample, is $358 \mathrm{~ms}$. A sweep cycle, lasting $3 \mathrm{~min}$, is typically comprised of 64 frequency measurements, each made during one spacecraft spin period. Thus a total of $8 \times 64$ voltage spectral density measurements received by each of the S, S', and Z antennae, as well as the corresponding times of measurement, are supplied for a single sweep cycle. Each measurement is provided in units of $\mu V^{2} \mathrm{~Hz}^{-1}$ and the preamplifier and receiver gain values have been taken into account. As well as this, general data including spacecraft attitude parameters and indicators for the mode of operation of the RAD1 receiver are supplied with each sweep cycle. L2-level data for the RAD1 instrument are provided as $\leq 480 \times 3$ min sweep cycles that comprise $24 \mathrm{hr}$ of RAD1 observations.

\subsection{Source Flux Density Determination and Calibration}

\subsubsection{Radio Background}

The frequency range of the RAD1 receiver is such that background radio emission is present due to both the local plasma environment and non-thermal emission from the galactic center or disk. At lower frequencies (below $\sim 300 \mathrm{kHz}$ ), the thermal motion of charged particles in the plasma surrounding the spacecraft creates QTN (Meyer-Vernet \& Perche, 1989) while emission from the galaxy dominates at higher frequencies (Cane, 1979; Novaco \& Brown, 1978). Previous examination of measurements across the entire WAVES frequency range has consolidated the measured galactic spectrum with previously derived functional forms (Dulk et al., 2001) and more recent measurements across the RAD1 receiver show agreement with a spectrum that falls off between 100 and $200 \mathrm{kHz}$ (Hillan et al., 2010). Hillan et al. (2010) produced a complete background spectrum, combining models of both the galactic background and a two-component model of the QTN that defines the signal above and below the plasma frequency. As well as these sources of emission, instrumental noise is also present due to the electronics of the antenna system and the digitization of the signal and can be derived from antenna-predeployment measurements; the galactic background (taken from a quiet period above $500 \mathrm{kHz}$ ) is no more than double the median RAD1 receiver noise. We assume that QTN at frequencies below $100 \mathrm{kHz}$ will typically dominate the background spectrum. A similar method to that used by Zarka et al. (2004) to determine the galactic background present in the Radio and Plasma Wave Science (RPWS) instrument of the Cassini spacecraft is implemented here for all frequencies. A background spectrum is formed for every 24 -hr period of RAD1 data by taking the $5 \%$ quantile at each frequency channel. Although no explicitly quiet period is selected over which to take the quantile (as opposed to the method of Zarka et al., 2004), the definition of the quantile imposes that the remaining 95\% of received signal is above this level. Although some examples of L2 data contain consistently high emission for the corresponding day at many frequencies, background spectra produced in this way agree well with other measured background levels observed by Wind, as well as producing the expected form due to the QTN and galactic background source described previously (Hillan et al., 2010). Once the background spectrum 
has been determined, the relevant value is subtracted from each of the eight measurements made during a spin period, implicitly assuming isotropy of the background source. If the data are negative, so unphysical, following background subtraction, the background value at the given frequency is stored instead.

\subsubsection{Calibration}

To relate the received power of the WAVES instrument to the AKR flux density, we consider the GP technique developed by Manning and Fainberg (1980). GP techniques allow for radio source parameters to be determined by inverse modeling the observations to the radio source parameters using the antenna reference frame and a model of the emission geometry. In their work, spin measurements from a synthetic inclined dipole (here fulfilled by the WAVES $S$ antenna) and a phase-shifted inclined dipole ( $S^{\prime}$ ) antenna are demodulated and combined with a spin-axis-aligned $(\mathrm{Z})$ antenna to derive the Stokes parameters (flux density and the degrees of linear and circular polarization), angular coordinates, and the angular radius of the source, so describing the state of a partially polarized extended source. To derive these parameters, it is assumed that a single radio source is observed by the instrument and that the source parameters are constant as the spacecraft completes a spin such that the modulation pattern can be inverted. For AKR this assumption is often broken; either the intensity of the source is variable on timescales lower than that of the spin or the spacecraft is observing emission from multiple sources in each measurement as it changes position during a spin or a combination of the two (see Section 2.3). It is not possible to use the combination of the WAVES antennae in this case as the exact variability over the modulation pattern cannot be determined analytically without a priori knowledge of the source parameters. However, the spin-axis-aligned Z antenna can be used to determine the source flux density after modifications are made to the original GP inversion. We assume that, for an AKR observation, the radio source is a point source and there is no linear polarization. We also assume that the AKR will be circularly polarized, but the received $\mathrm{Z}$ antenna power is independent of the source circular polarization using the inversion of Manning and Fainberg (1980). Following from Equation 21 in Manning and Fainberg (1980), this gives

$$
P_{Z}=\frac{1}{2} G S_{Z} \sin ^{2} \theta
$$

where $P_{Z}$ is the power measured by the $\mathrm{Z}$ antenna in $\mathrm{V}^{2} \mathrm{~Hz}^{-1}, G$ is a calibration factor, $S_{Z}$ is the flux of a point source derived from the single $\mathrm{Z}$ antenna in $\mathrm{Wm}^{-2} \mathrm{~Hz}^{-1}$ and $\theta$ is the co-latitude of the radio source. For AKR, we can assume that the source region is approximated by the Earth's center and transform the source co-latitude $\theta$ to $\lambda$, the latitude of the spacecraft in geocentric-solar-ecliptic (GSE) coordinates. Given that the calibration is applied to all Wind observations during the interval, which are made from various latitudes and LT, and that we cannot determine the emission hemisphere exactly, this assumption is most broadly valid; errors in the spatial location of the source when assuming emission from either pole could be up to $1 R_{E}$ given the spectral range of the instrument, the azimuthal extent of AKR sources relative to Wind and their confinement along high-latitude magnetic field lines. We have characterized the error due to assuming the Earth's center as the source location instead of the geographic poles and found that the maximum error implicit for this period is 1.2\%. Details of this statistical error analysis are given in Appendix A. This modification gives

$$
P_{Z}=\frac{1}{2} G S_{Z} \cos ^{2} \lambda
$$

as the received signal of the $\mathrm{Z}$ antenna, oriented normal to the ecliptic plane, is minimized when the spacecraft is above the poles. This then gives the flux density $S_{Z}$ for each of the eight spin measurements, which are then averaged using the mean.

The synthetically inclined $\mathrm{S}$ (and phase-shifted S') antenna is longer and more sensitive than the $\mathrm{Z}$ antenna, making its measurements prone to spurious signals at all frequencies as receiver electronics are saturated. This is often the case when Wind observes the highly intense AKR, which for this study occurs mostly when the spacecraft is near perigee. This contamination does not occur with the shorter, less sensitive $Z$ antenna. While the $Z$ antenna is less prone to saturation, its lower sensitivity means that previous methods of calibration with Wind/WAVES cannot be used. These have employed measurements of the galactic background to determine a value of the instrument gain, using a model of the galactic emission to infer the observed flux during a quiet period and then equate this to measurements (Zarka et al., 2004; Zaslavsky et al., 2011). The shorter $\mathrm{Z}$ antenna typically measures the galactic background to be within $10 \mathrm{~dB}$ of the instrumental noise, 
as given by the received power prior to antenna deployment, so the contribution from the galactic signal cannot be determined. For this reason, the initial measurements of the instrumental characteristics are used to determine the gain using

$$
G=\left(\frac{C_{a}}{C_{a}+C_{s}}\right)^{2} L_{e f f}^{2} Z_{0}
$$

where $Z_{0} \sim 120 \pi$ is the impedance of free space, $C_{a}=30 \mathrm{pF}$ and $C_{s}=45 \mathrm{pF}$ are the antenna and stray capacitances, respectively, and $L_{e f f}$ is the effective length of the antenna. Here, we use the physical length $\left(L_{p}=4.65 \mathrm{~m}\right)$ of each monopole of the $\mathrm{Z}$ antenna, given that both electrical monopoles that comprise the $\mathrm{Z}$ antenna are in the short-dipole regime, so that $L_{e f f}=L_{p}$.

To check the validity of using calibrated measurements from a linear antenna as proxy for the true flux of a radio source (here AKR), we compared observations of solar radio Type III bursts from Wind and Cassini. A modified calibration routine was prepared and applied to the linear $\mathrm{Z}$ antenna for these observations. We compare the flux with independent values produced by a direction-finding inversion, derived from the same initial method presented by Manning and Fainberg (1980) but bespoke to solar Type III burst observations with Wind, giving the flux density, angular coordinates and angular extent of the source with only the $\mathrm{S}$ and $\mathrm{Z}$ antennae. Initially, these independent measurements served as a scaling factor to be applied to $S_{Z}$ from Equation 2, using an average spectrum of peak fluxes across a set of Type III bursts. After discussion during the review process, it became clear that this scaling factor is only relevant for Type III bursts, but not for AKR, since it essentially corrects for the size of an extended radio source. AKR can be considered as coming from a point-source when observed at large radial distances, which is why we present $S_{Z}$ as an accurate (within instrumental uncertainties in the terms of Equation 3) measure of the AKR flux. Appendix B gives explicit details of this former processing step and is retained for the interest of the reader.

\subsection{Empirical Selection of AKR}

As mentioned in Section 2.1, the Wind/WAVES $\mathrm{Z}$ antenna is spin-axis aligned. As the spacecraft rotates, antennae in the spin plane observe a modulating signal that is dictated by the rotation period and the position of the antennae with respect to the source location and other parameters. While this modulation can be used to derive source parameters using GP inversion as previously mentioned, these require that the source parameters are constant during a spacecraft rotation; a constraint which is often broken for AKR. The spin-axis aligned $\mathrm{Z}$ antenna sees no such modulation due to the spacecraft rotation, and so any variability in the received power can be attributed to changes in the intensity of a radio source, assuming that a single source is observed. It has been mentioned that the spacecraft measures a superposition of multiple spatially separated sources, limiting the observations by the temporal and spectral resolution of the instrument. Although this is less true for the spin period than it is for the sweep duration, it is possible that the variability measured by the $\mathrm{Z}$ antenna for a spin is due to the more slowly varying intensity of separated sources. Given the generation mechanism of AKR, however, it is likely that a single source will have a highly varying amplitude on spin timescales of seconds. While it is nontrivial to discern between the two effects, it is important to note that this may also lead to an overestimation of the AKR selection, although this effect is negligible when data are averaged over the sweep duration.

A statistical proxy for AKR can be derived from the $\mathrm{Z}$ antenna by modeling the 8 measurements made during a single spin as a normal distribution centered on a mean intensity, with any variability then being described by its standard deviation $\sigma$. To be able to compare the standard deviation between sources of different mean intensities, the measurements are normalized by the mean intensity of the spin. This gives $\sigma_{Z}$, the standard deviation of the spin-normalized $\mathrm{Z}$ antenna measurements. $\sigma_{Z}$ is calculated prior to background subtraction, to retain the observed variability (it is assumed that any variability represented by $\sigma_{Z}$ will be predominantly influenced by the sources of the strongest intensity). Figure 1a shows the spin-normalized measurements made by the $\mathrm{S}$ and $\mathrm{Z}$ antennae during an exemplary AKR burst. It is not clear from the figure that the $S$ antenna is displaying an insufficient modulation pattern to derive the GP source parameters, and an analytic relationship between the $\mathrm{S}$ and $\mathrm{Z}$ measurements is not known. However, the variability across the $\mathrm{Z}$ antenna measurements shows that the observations do not meet the criteria of constant intensity for GP inversion, and the $\sigma_{Z}$ value for this spin is given in the legend of the bottom panel of Figure 1a. Figure 1b 


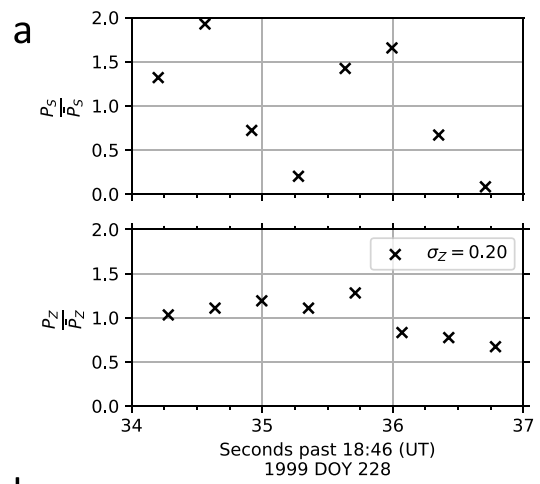

b

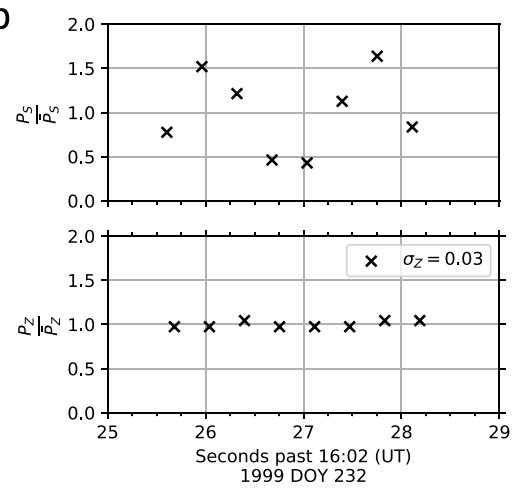

C
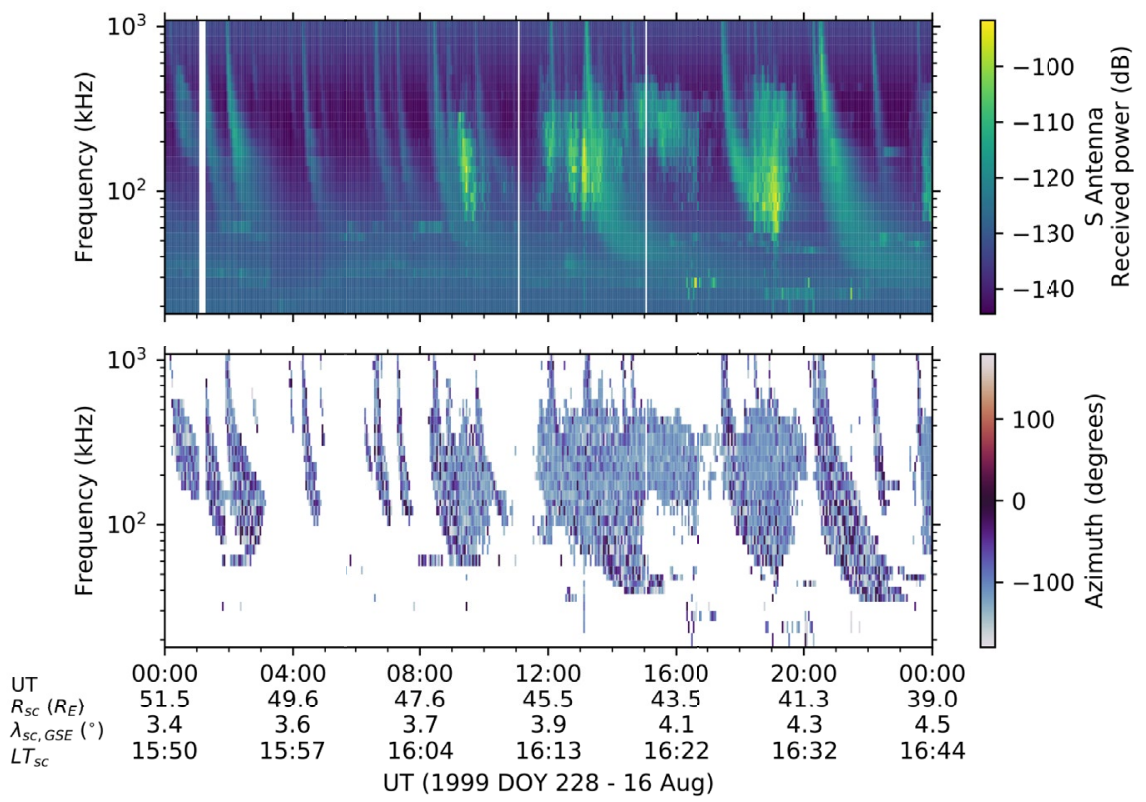

Figure 1. Measurements $\left(P_{S}\right.$, and $\left.P_{Z}\right)$ made by the synthetic-inclined (S) and spin-axis-aligned $(\mathrm{Z})$ antennae of Wind/WAVES during a single spin, normalized by the average received power during this time $\left(\bar{P}_{S}, \bar{P}_{Z}\right)$. (a) Spin observations at $124 \mathrm{kHz}$, corresponding to a rotation during the burst of Auroral Kilometric Radiation (AKR) emission between roughly 1800 and 2000 UT in panel (c). (b) Spin observations of an isolated Type III burst at $124 \mathrm{kHz}$, on 1999 day of year (DOY) 232, illustrating the modulation pattern produced in the $\mathrm{S}$ antenna and the lack thereof in the $\mathrm{Z}$ antenna, observed for measurements of a near-constant intensity source. The value of the selection metric, $\sigma_{Z}$, is given in the legend of the bottom panel for both (a) and (b). (c) Dynamic spectrograms showing $24 \mathrm{hr}$ (1999 DOY 228) of the power received by the $\mathrm{S}$ antenna, in $\mathrm{dB}$, in the top panel. Only observations with a signal-to-noise ratio (SNR) of $>25 \mathrm{~dB}$ are shown, for clarity. The bottom panel shows the apparent azimuth of the radio source, determined by the application of a Goniopolarimetric (GP) inversion for use with Wind AKR observations. Only observations with a signal-to-noise ratio (SNR) above $25 \mathrm{~dB}$ are shown. The azimuthal angle of the source is given in a nonrotating coordinate system (see text) with angles of $0^{\circ}$ indicating a source in the direction of the Sun, negative angles indicating a source to the right of the Wind-Sun line and positive angles indicating a source to the left of the Wind-Sun line. For the period shown in panel (c), Earth is at approximately $-130^{\circ}$ in this coordinate system.

shows similar data for an observation of a solar radio Type III burst, with the double-peaked modulation pattern in the received power clearly visible in the top panel and the $\mathrm{Z}$ antenna measuring nearly constant intensity emission; illustrating measurements of a radio source more appropriate for use with a GP inversion. While a comprehensive study of the $\sigma_{Z}$ distribution is not included, examination of the dynamic spectrograms show that the $\mathrm{Z}$ antenna consistently measures higher variability (using $\sigma_{Z}$ ) during periods of AKR bursts. To select regions that correspond to AKR emission, a numerical threshold is chosen based on the visual identification of the dynamic spectrograms from Wind during the Cassini flyby; here $\sigma_{z} \geq 0.1$. Given that the opposite limit of $\sigma_{Z}$ selects sources that are less variable, $\sigma_{Z}$ could be useful to select data that are more appropriate for a GP inversion with Wind. See Appendix $C$ for further justification of the choice of the threshold value.

Figure 1c shows $24 \mathrm{hr}$ dynamic spectrograms of Wind AKR observations and their general unsuitability for GP applications. The top panel shows a spectrogram of the measurements made by the S antenna; relatively faint Type III bursts are seen throughout the day, whilst bursts of intense AKR are also observed. The bottom panel shows the result of an attempt to apply the GP inversion of Manning and Fainberg (1980) to the Wind observations. The azimuthal angle of the source is given in a fixed coordinate system, independent of the spacecraft rotation that is defined by an $\mathrm{x}$ axis in the direction of the Sun with the $\mathrm{x}$ - and $\mathrm{y}$ axes in the ecliptic plane, and a $\mathrm{z}$ axis that completes the orthonormal frame and is directed Southward of the ecliptic plane. Given that the retrieval of direction-finding parameters for radio sources is dependent on a strong signal, only observations with a signal-to-noise ratio (SNR) of $>25 \mathrm{~dB}$ are shown, highlighting the presence 

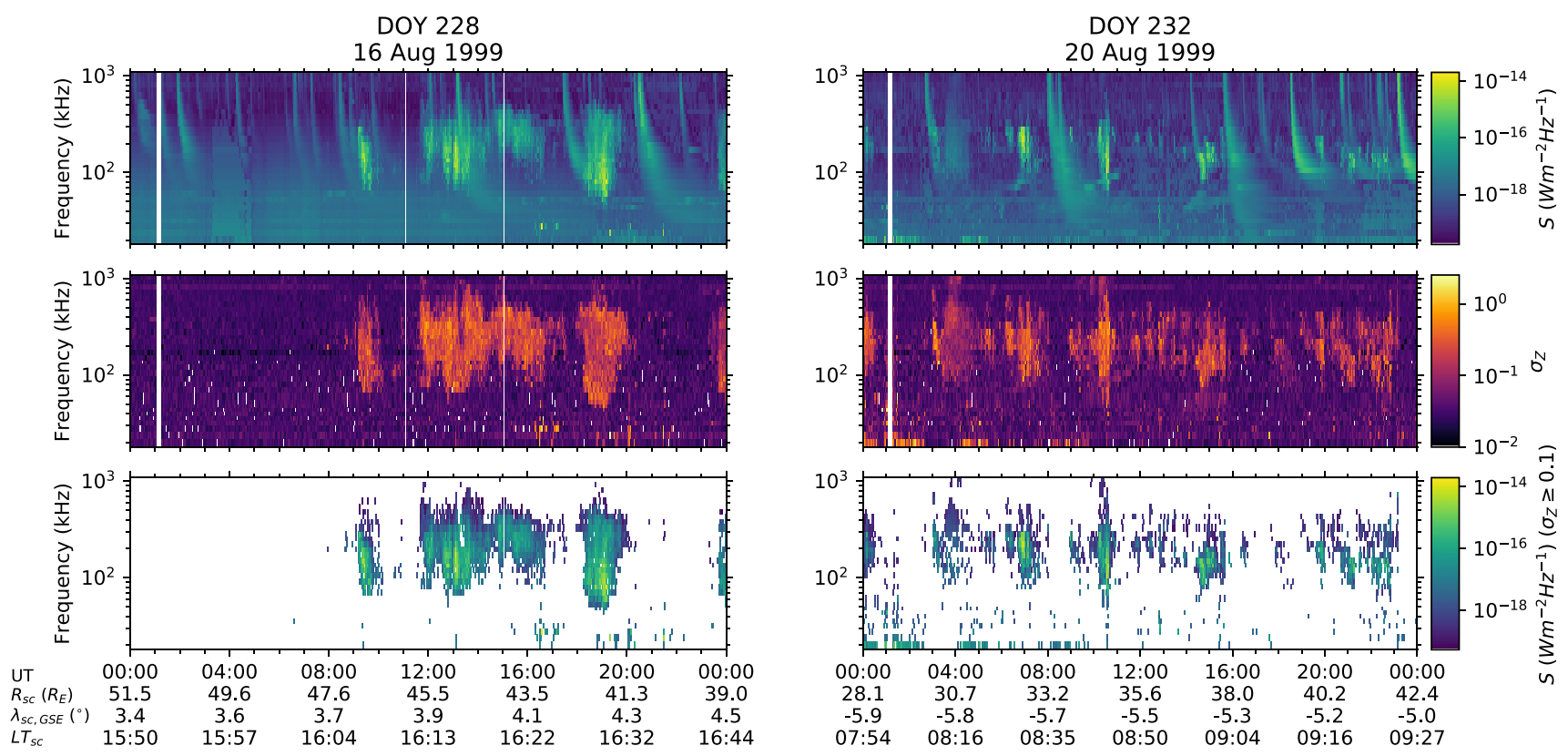

Figure 2. Two examples of application of the empirical selection detailed in Section 2.3 to $24 \mathrm{hr}$ of Wind data (3- min resolution). For each example of panel (a) (left) and panel (b) (right), the top panel shows flux density observed by Wind and derived by the method outlined in Section 2.2, the middle panel shows $\sigma_{Z}$, the statistical proxy of the source amplitude variability, and the bottom panel shows the flux density with the selection mask applied (see Section 2.3). The lower limit of the color bar of the middle panel is set at $10^{-2}$ for visual clarity. The radial distance, ecliptic latitude, and local time for each example are shown in the ephemeris at the bottom of the plot.

of solar radio Type III bursts (multiple examples between $\sim 00-1,000 \mathrm{MHz}, 0000-0400 \mathrm{UT}$, or at all frequencies after 2000 UT) as well as AKR. Azimuthal angles of $0^{\circ}$ indicate a source in the direction of the Sun, negative angles indicate a source to the right of the Wind-Sun line, and positive angles indicate a source to the left of the Wind-Sun line. The GP inversion is subject to a degeneracy in the wave vector direction, so angles that suggest an unphysical source direction for AKR have been transformed appropriately; Earth is at approximately $130^{\circ}$ for the data shown. For the AKR between 0800 and 2000 UT, the azimuthal angle is poorly constrained and has high variability during AKR observations due to the aforementioned variability during the spacecraft rotation. While $\sigma_{Z}$ acts as a proxy for the radio source here, we note that without access to the GP inversion and polarization information, we cannot make an exact physical inference and so the selection is empirical. With average $\sigma_{Z}$ spectra for each sweep, a mask can then be created and applied to the calibrated flux densities to select data that meet this criteria; here the flux densities are also averaged across the 3- min sweep cycle. In this case, the consideration of multiple spatially separated sources cannot be ignored, and the flux spectra represent the spatial average of AKR emission across a relatively wide longitude as well as a temporal average.

Figure 2 shows two examples, (a) and (b), of $24 \mathrm{hr}$ of calibrated Wind/WAVES data (top panel) with the variability proxy $\sigma_{Z}$ (middle panel) and the application of the resulting mask (bottom panel). Wind observations in both (a) and (b) contain many Solar Type III bursts with various drift rates that at times cover the entire RAD1 frequency range, as well as increases in QTN and emission at the plasma frequency at frequencies $<100 \mathrm{kHz}$. The middle panel shows the effectiveness of $\sigma_{Z}$ as an identifier of AKR emission, with observations of Type III bursts exhibiting a standard deviation less than that of the AKR by about an order of magnitude. AKR is generally more intense than Type III bursts, and this couples with the aforementioned considerations of the AKR generation mechanism and viewing geometry to produce the observed discrepancy in the $\sigma_{Z}$ distributions of each source, as shown here by the middle panel of Figure 2. While not shown here, the $52 \mathrm{kHz}$ channel has persistently higher $\sigma_{Z}$ due to radio frequency interference (RFI). In Figure 2 (and Figure 5), the $52 \mathrm{kHz}$ channel has been removed and the values of $\sigma_{Z}$ in neighboring channels used to interpolate an updated "background" value to increase visual clarity. For the remaining analysis, after selecting the AKR data with the $\sigma_{Z}$ criterion, flux densities from the $52 \mathrm{kHz}$ channel are removed to avoid contamination. AKR can be seen in both examples; in (a), Wind is approaching perigee at around 


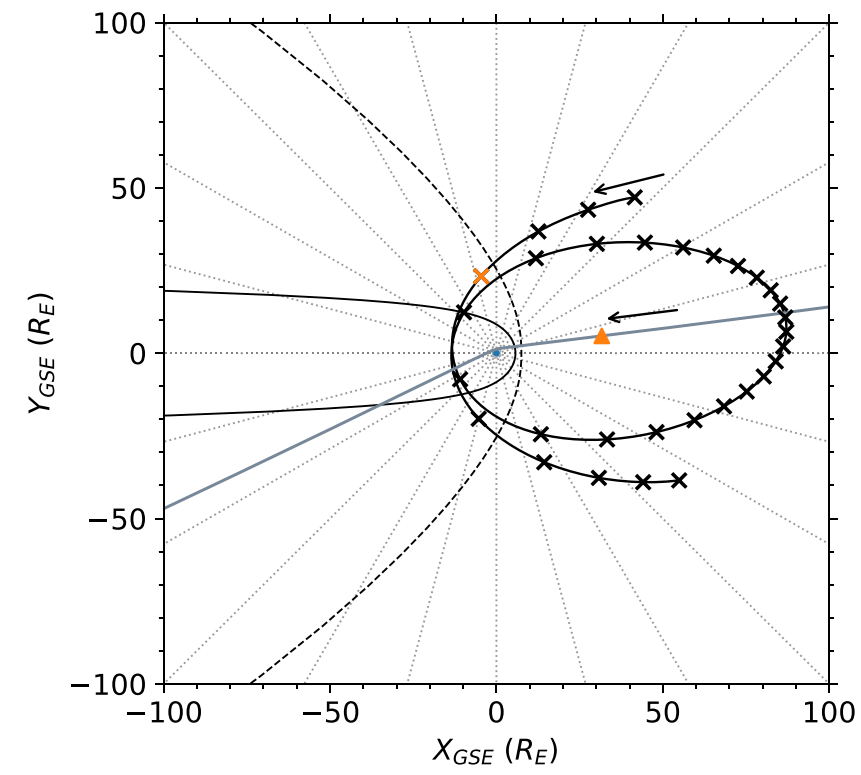

Figure 3. Orbital trajectory of Wind for 1999 day of year (DOY) 227-257, projected onto the ecliptic plane. The start of each day for the Wind trajectory is marked by crosses. Also shown in gray is the Cassini trajectory for the closest approach, with the start of DOY 230 marked by the triangle. The Cassini trajectory can be found to a greater extent in figure 2a of Lamy et al. (2010). The arrows indicate the direction of travel for both spacecraft and the colored markers indicate the start of DOY 230. Average modeled magnetopause and bowshock surfaces are shown as thin solid and dashed lines, respectively; the Sun is to the right. The former is given by the model of Shue et al. (1998), using solar wind data from OMNI with average parameters. The latter surface is derived using similar data and the model of Wu et al. (2000).
1600-1700 LT and $4^{\circ}$ latitude, and emission is observed for $11 \mathrm{hr}$ between $\sim 80-800 \mathrm{kHz}$; it exits perigee in (b), crossing 0800-0900 LT around $-6^{\circ}$ latitude and observing more sporadic emission patches throughout the day across a narrower frequency range, $\sim 100-500 \mathrm{kHz}$.

Given that the metric for selection, $\sigma_{Z}$ is determined using the variance of measurements made during a spin, there are other sources of emission that could be responsible for the retention of a particular sample. Instrumental RFI is an example of this, but particularly rapid changes in intensity due to energetic fluctuations in the local plasma can also produce signatures similar to those produced by AKR. Particularly when Wind travels through the turbulent, dense plasma in the magnetosheath (see DOY 229-231 and 251-254 in Figure 5), the majority of the emission at lower frequencies is retained. At times when Wind is on the dayside, within the solar wind, emission is occasionally selected that has no observed, corresponding AKR signal at higher frequencies and is assumed to be caused by similar local and temporal variations in the plasma. Intensification of the signal at other characteristic frequencies of the plasma are also occasionally seen and retained by the selection, such as emission at the plasma frequency. The retention of non-AKR signals is lessened at higher frequencies, where Wind typically sees contributions from AKR, Jovian hectometric or kilometric radiation, which is often faint (Zarka et al., 2004), or solar radio bursts that have little variability across the spacecraft spin period. As well as other sources of emission that could be responsible for the variability, there is also the possibility that Wind is subject to incident waves from multiple radio sources. Due to this, it is not guaranteed that only AKR will be present in any of the selected data, although the most intense emission determines the modulation pattern in these cases. AKR is typically more intense than other radio sources for Wind observations, and this is the case particularly when Wind is at a favorable viewing position for AKR. Observations where solar radio type III bursts are the most intense emission arriving at the $\mathrm{Z}$ antenna, for example, are thus removed using the selection with $\sigma_{Z}$. Although fine structure and variability has been observed in the spectra of Type III bursts and there is no complete way to discriminate between this variability and that exhibited during AKR observations, applications of the selection such as those in Figure 2 show that, on average, observations where the Type III dominates are removed. Given the ubiquity of Type III bursts and sources of other less variable emission and the simplicity of the $\sigma_{Z}$ selection criteria used here, the technique is utile for studying AKR emission, as shown in the following section and conversely for removing the contribution of AKR in studies of solar radio bursts. Applying the flux-density calibration and selection of AKR to Wind/WAVES observations since 1994 can form a unique data set to investigate AKR characteristics. The next section takes advantage of the selection to make an initial comparison to AKR radio measurements obtained by Cassini in 1999.

\section{Application to Wind/WAVES for Multipoint AKR Observations With Cassini}

\subsection{Spacecraft Ephemerides}

Figure 3 shows the Wind trajectory projected onto the ecliptic plane for the period studied here, as well as part of the Cassini trajectory. The start of day of year (DOY) 230 is indicated in color for each spacecraft, during which Cassini reached its closest approach to Earth at a radial distance of $1.2 R_{E}$. After traversing the dawn flank of the magnetosphere and passing Earth, Cassini continued downtail between 0100 and 0200 LT at $\sim 9 R_{E} / h$. Figure 4 shows the latitudes of both spacecraft in both geocentric solar ecliptic (GSE) and magnetic coordinates. The latter refers to the coordinate system used in Lamy et al. (2010), which defines positive latitudes as those in the Northern magnetic (Southern rotational) hemisphere. 

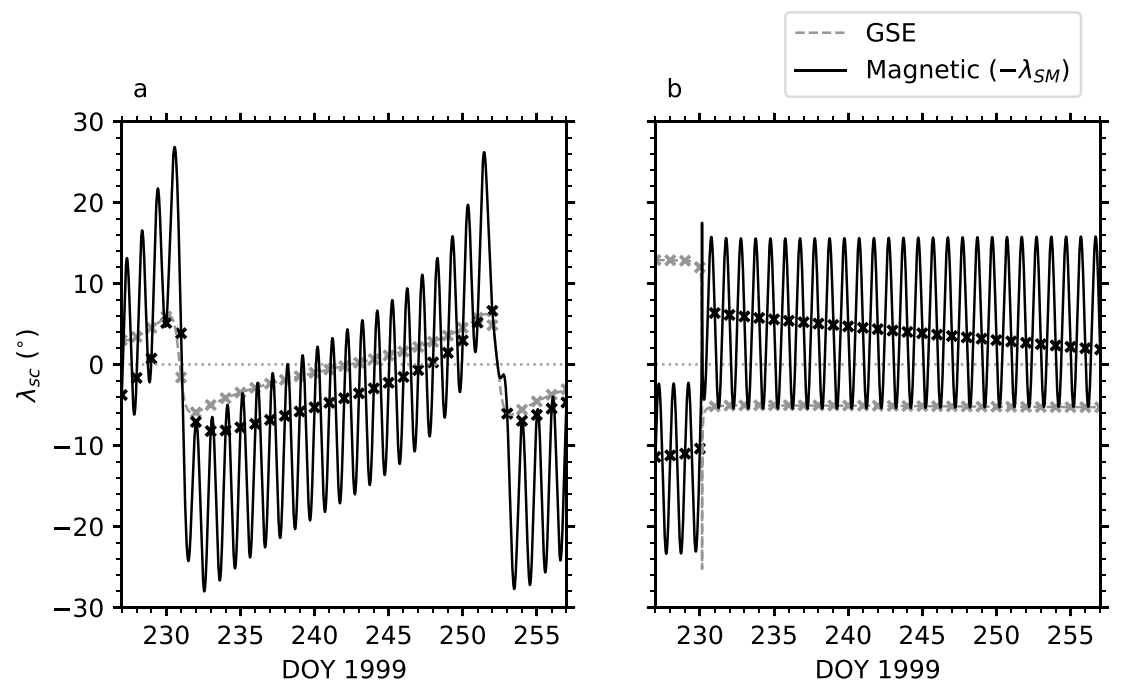

Figure 4. Latitudes of the Wind (a) and Cassini (b) spacecraft for the period shown in geocentric (GSE) coordinates with the gray, dashed line and magnetic coordinates with the black, solid line. Magnetic coordinates are as defined in Lamy et al. (2010), with positive magnetic latitudes in the Southern geographic (Northern magnetic) hemisphere. The magnetic coordinate system used here has the z-direction oppositely defined to that in solar magnetic (SM) coordinates, indicated by $-\lambda_{S M}$ in the legend. Markers show the beginning of each day.

During Cassini's flyby of Earth and the 30- day period studied by Lamy et al. (2010), from August 15 to September 14, 1999 (DOY 227-257), Wind completed close to two petal orbits with a perigee radii of $\sim 13 R_{E}$ and apogee radii of $\sim 88 R_{E}$. At the start of this period, Wind approached its first perigee from a position duskward of Earth at roughly 1500 LT on DOY 227, with a GSE latitude of $3.0^{\circ}$ and a radial distance of $67 R_{E}$. From there, Wind approached the magnetosphere and crossed the bow shock and magnetopause before the first perigee was reached. Wind reached perigee around $0100 \mathrm{LT}$, at a GSE latitude of $1^{\circ}$, while traversing the magnetotail. Wind then exited the magnetosphere, covering the dawn flank and reaching apogee on DOY 241 at a GSE latitude of $-0.4^{\circ}$ around 1200 LT. After 23 days, Wind reached 1500 LT once more at a closer radial distance of $44 R_{E}$ and a GSE latitude of $4.6^{\circ}$. Entering the nightside magnetosphere for the second time during the period, Wind reached a second perigee on DOY 252 at 0000 LT and close to the ecliptic plane. Wind then exited the magnetosphere once more, and the final observations that are conjunct with Cassini are made at around $1000 \mathrm{LT}$ with GSE latitude of $-3.0^{\circ}$ and a radial distance of $67 R_{E}$.

As mentioned in Section 1, GP inversions have been successfully applied to observations from Cassini and allowed the circular polarization of the radio emission to be determined and thus the hemisphere of origin. For the empirical selection of AKR emission used here with Wind, there is no unambiguous way to determine circular polarization from flux measurements. Due to the anisotropic, widely beamed emission from the AKR source regions, visibility of the emission from either pole is highly dependent on the magnetic latitude of Wind and inferences of the origin of the emission can be made based on this. While Wind is on the dayside, approximately between the two perigees at DOY 231 and DOY 252, it covers a range of low magnetic latitudes in first the Southern then the Northern magnetic hemispheres, crossing the magnetic equator near the apogee (see Figure 4). In both cases, where Wind crosses the nightside and where AKR is expected to be most visible, the spacecraft approaches from the dusk flank in the Northern magnetic hemisphere and crosses into the Southern magnetic hemisphere at perigee. Magnetic coordinates are as defined in Lamy et al. (2010), with positive magnetic latitudes in the Southern geographic (Northern magnetic) hemisphere. The magnetic coordinate system used here has the z-direction oppositely defined to that in solar magnetic (SM) coordinates. Given that the magnetic latitude does not exceed $30^{\circ}$, it is uncertain whether or not the spacecraft will be in either or both of the regions illuminated by emission from either hemisphere. Previous examination of the average AKR source region has suggested that emission from both hemispheres can be observed at distances $>12 R_{E}$ in the equatorial plane (Gallagher \& Gurnett, 1979), with the approximate perigee distance of Wind $\left(\sim 13 R_{E}\right)$ implying that this will be the case for all nightside observations for this period. In their study, Lamy et al. (2010) use the polarisation information to assess the average AKR power 

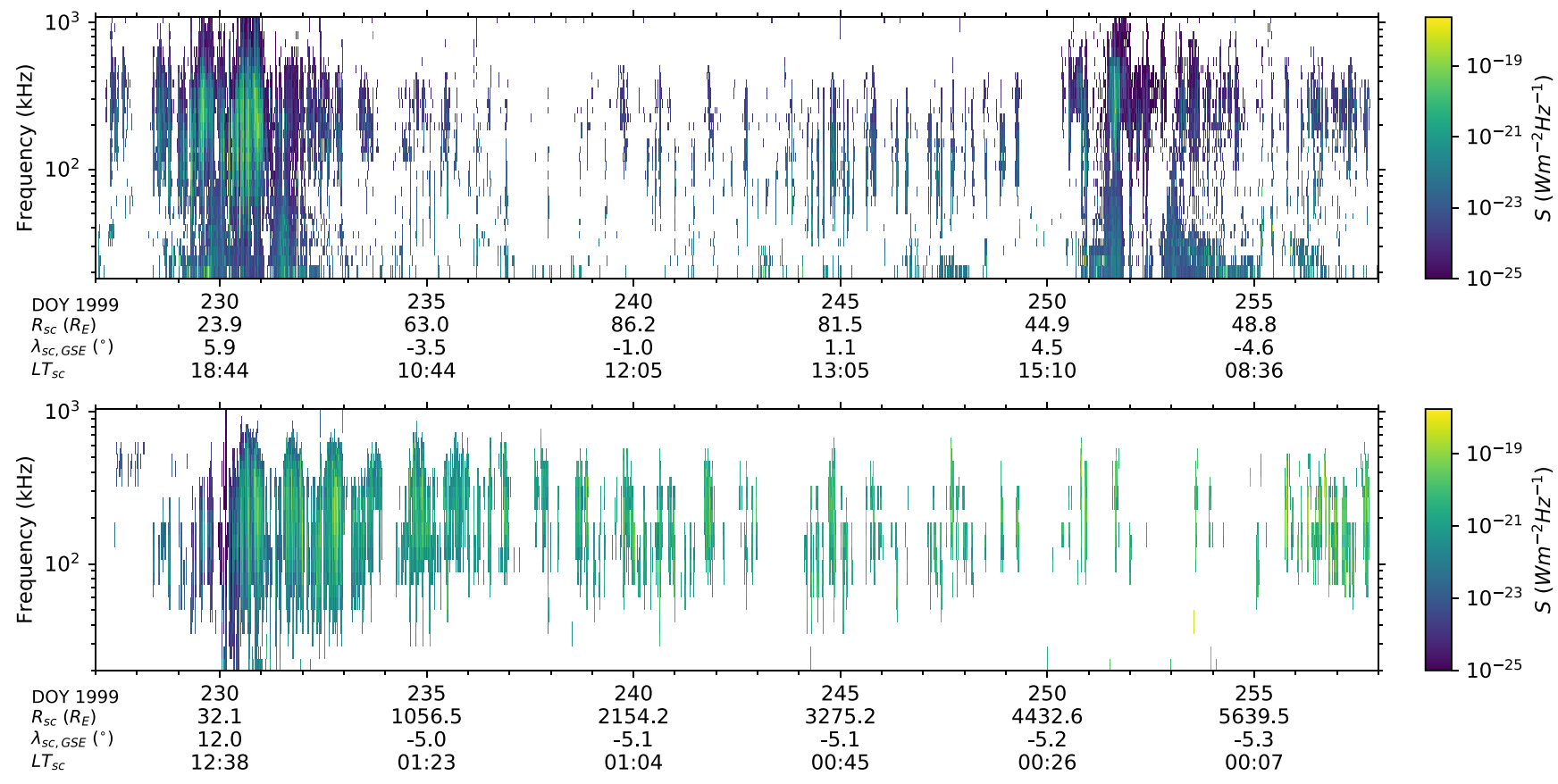

Figure 5. Dynamic spectrogram showing the flux density measured by Wind/WAVES (top) and Cassini (bottom) for 1999 day of year (DOY) $227-257$ and normalized to a distance of $1 \mathrm{AU}$. The top panel shows the average flux density at a 3- min resolution as selected with the $\sigma_{Z}$ threshold described in Section 2.3. The flux density is computed by calibrating the power received by the spin-axis aligned $\mathrm{Z}$ antenna, as outlined in Section 2.2.2. The bottom panel shows the flux density observed by the Cassini spacecraft, namely the maximum of the left-handed circularly polarized or right-handed circularly polarized Auroral Kilometric Radiation (AKR) emission of a given frequency at a 90- s resolution (for complete details of the calibration and selection of AKR with Cassini, see Lamy et al., 2010). The radial distance, ecliptic latitude, and local time of each spacecraft are shown in the ephemeris at the bottom of each panel.

from each hemisphere when Cassini is above a given magnetic latitude finding that LH AKR was close to 4 orders of magnitude weaker than the RH AKR when the spacecraft had a magnetic latitude $\lambda_{m}>10^{\circ}$. This suggests that Wind is likely illuminated by emission from AKR sources in both hemispheres whilst near the equatorial plane, with Southern, LH emission likely to be dominant prior to perigee and Northern, RH emission afterward.

\subsection{AKR Flux Density and Power}

Figure 5 shows the AKR flux density of both Wind and Cassini for the entire 30- day period studied here. The AKR flux density with Wind is obtained using the calibration and selection outlined in Section 2. The AKR flux density with Cassini contains that of both LH- and RH-circularly polarized AKR, obtained using a GP inversion technique and selecting data with $|V| \geq 0.2$, where $V$ is the normalized Stokes parameter describing circular polarization (Lamy et al., 2010). The maximum of either the LH or RH AKR is shown at $90 \mathrm{~s}$ resolution in the Cassini spectrogram. Both flux densities are scaled for the distance from the approximate source (Earth's center) and normalized to $1 \mathrm{AU}$ to enable a comparison between the two data sets. The general effect of viewing position on the AKR observations from each spacecraft can be seen here; emission is stronger and more consistent for Cassini as it travels away from Earth downtail, remaining on the nightside, while a periodic variability is seen in the Wind spectrogram as it passes the nightside during its perigees. While the day-to-day variability in the observed emission is not studied here, it is interesting to note differences between the two perigee observations. Wind sees more persistent, stronger emission for the first perigee ( DOY 229) than the second ( DOY 251 ). While this variability between orbits is not surprising, given the changing solar wind conditions that affect AKR, it demonstrates the differences between observations made at different times, which will clearly bias any average result with a limited selection of data.

Figure 6 shows AKR flux density spectra from both spacecraft given by statistical thresholds, namely the median spectra and those for the $90 \%$ and $99 \%$ quantiles or increasing intensity thresholds. The top panel shows Cassini flux densities for both LH and RH circularly polarized AKR, reproducing figure 6a of 

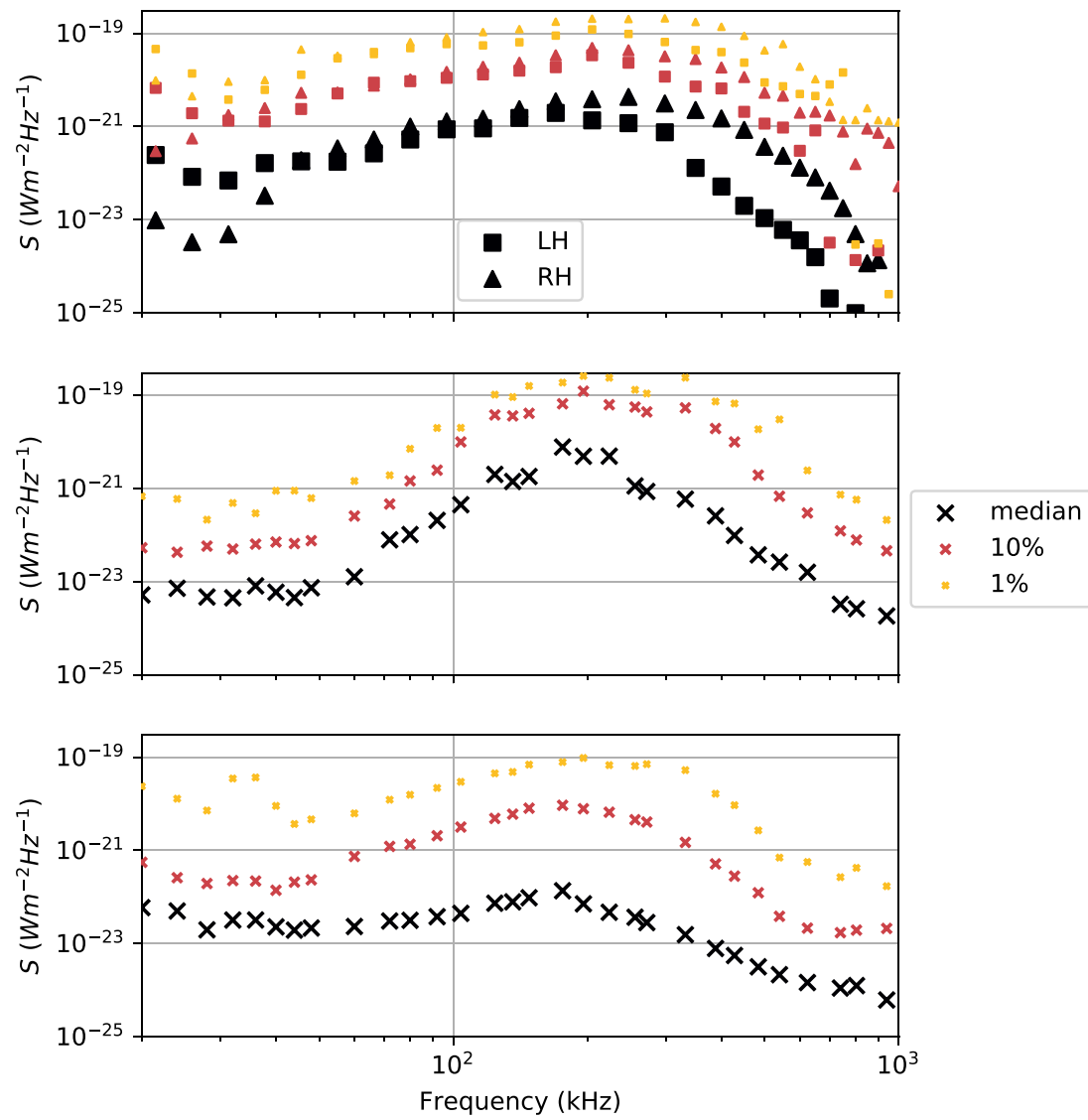

Figure 6. Reduced flux density spectra comparing Auroral Kilometric Radiation (AKR) observations from Cassini during the whole period (top panel) with those made by Wind when in a comparable viewing position (middle and bottom panels). For this, the Wind data were further selected such that the spacecraft was located from $-23^{\circ} \leq \lambda_{m} \leq 16^{\circ}$ and within $0000-0200 \mathrm{hr}$ LT. Flux density data in each case are reduced to give the measured intensity that reached 50\% (median - black), 10\% (red), and 1\% (orange) of the time. The top panel shows spectra for both the LH and RH circularly polarized AKR as given by the GP inversion applied to Cassini. The middle panel shows AKR- and position-selected data from Wind during the 30-day period studied here. The bottom panel shows AKR-and position-selected data from Wind for all of 1999, to increase the statistical rigor of the selection verification (see main text).

Lamy et al. (2010). The middle panel shows Wind flux densities returned by the selection (described in Section 2.3) applied to data from 1999 DOY 227-257. Given the anisotropy of the AKR beaming and the longitudinal distribution of AKR source regions, it cannot be assumed that each spacecraft will observe the same emission region at a given time from different viewing positions in space. To elicit a valid comparison of the reduced spectra between the spacecraft, we select Wind data such that only observations made from a similar viewing position are included; Cassini was in the region with a magnetic latitude $-23^{\circ} \leq \lambda_{m} \leq 16^{\circ}$ and within 0000-0200 hr LT during its flyby. A complete discussion of the flux densities observed by Cassini can be found in Lamy et al. (2010), but here we compare the main features with those of Wind.

While the Cassini spectra for both LH and RH AKR fluxes see a mostly shallow increase up to the peak at $\sim 200 \mathrm{kHz}$, this is not the case for Wind. A consistent plateau is seen in each of the spectra in the middle panel below $50 \mathrm{kHz}$, after which the flux density increases more sharply for each quantile. Discrepancies in the spectra can be seen at lower frequencies, which is likely due to a combination of the nature of low frequency AKR emission and the fragmented viewing by Wind when in the appropriate position. While Cassini makes 30 days of remote observations in this magnetic latitude and LT range, Wind spends $\sim 9 \mathrm{hr}$ in the same position, with each observation $\sim 20 \mathrm{hr}$ apart. Without considering the viewing effects, the transience of AKR below $100 \mathrm{kHz}$ alone could produce the discrepancies in the average spectra. As well as this, Wind is closer Earth and passes the nightside while crossing the magnetic equator, which could affect 
the viewing of high altitude, low frequency AKR sources. The $\sigma_{Z}$ selection is limited at low frequencies due to contamination by QTN and local plasma waves; although we assume that the aforementioned effect dominates, given that Wind is likely inside the magnetosphere for the selected observations (as shown by the average magnetopause boundary in Figure 3), where these emissions have less of a presence than in other regions. Figure 6 also shows better agreement between the two spacecraft at $>100 \mathrm{kHz}$, the resulting range including the typical spectral peak of AKR. The AKR emission at higher frequencies is known to be more temporally consistent and can be said to be better representative of the average AKR signal compared between remotely observing spacecraft. For these reasons, in the following, only the selected signal above $100 \mathrm{kHz}$ is considered. At frequencies higher than this, discrepancies between the spectra may exist simply due to the two spacecraft primarily observing different AKR source regions as we do not expect the AKR spectrum to be constant at all LT.

Again comparing the Wind spectra in the middle panel with Cassini in the top panel of Figure 6, the peak of the Wind median spectrum agrees, existing at a frequency close to $200 \mathrm{kHz}$ and between the peak flux of the Cassini LH and RH AKR median spectra; also in agreement with initial AKR observations (Kaiser \& Alexander, 1977). While the differences at lower frequencies are likely due to contamination, the Wind median spectrum falls off more rapidly than the $\mathrm{RH}$ median spectrum of Cassini and is more comparable to the LH median spectrum. At frequencies $>700 \mathrm{kHz}$, the Wind median spectrum is more closely comparable to the RH AKR median spectrum, although the aforementioned limitations prevent close physical interpretation of the differences or similarities between the spectra. Generally, however, the Wind median spectrum has a minimum at the highest observed frequencies, which again agrees with the Cassini measurements. Each of the Wind spectra that show the higher intensity thresholds in the middle panel show generally good agreement in magnitude with those of Cassini; a similar increase of 2 orders of magnitude between the median and highest intensity spectra is seen in the selected Wind data. This is interesting considering the small amount of time Wind spent in the region relative to Cassini and suggests that the limited Wind measurements here are characteristic of the AKR that Cassini observes for the whole period. There is also evidence of broadening of the spectral peaks to higher frequencies with increasing intensity in the middle panel as observed by Cassini.

Given that Wind spends the least amount of time on the nightside during perigee for this period, it is important to consider the limiting effect of the position selection. To highlight this, while Wind spends $\sim 1.2 \%$ of the time in the specified region during this 30- day period, 5.1\% of the AKR data selected here is observed in this region. However, this increase shows the efficacy of the empirical selection in reflecting the preferential location of the nightside for observing AKR emission and the AKR sources themselves. Although we cannot compare the Cassini spectra with Wind observations made outside of the temporal range covered by the Cassini flyby, increasing the scope of the data included can allow us to characterize the selected data more rigorously by comparing the general features of the spectra. For this reason, the bottom panel of Figure 6 shows spectra defined by the same thresholds but applied to Wind data from the entirety of 1999 after selecting the AKR as described. Although the magnitude of the spectra is lower (which is expected as more AKR emission is included, assuming that the more extreme events will happen less regularly), the broadening of the spectral peak to higher frequencies with increasing intensity is present. There is a larger increase of 3 orders of magnitude between the median and highest quantile spectra in the bottom panel, suggesting that the observations made in the 30- day period here are more intense than other times in the same year. There is also a separate, much shorter peak around $30-40 \mathrm{kHz}$ that exists in the highest intensity spectra of the bottom panel, which could be indicative of the average state of the magnetosphere or solar wind throughout 1999 as consistent Langmuir wave excitations may be seen close to the local plasma frequency in this region.

The AKR source region, as discussed in Section 1, is typically found at altitudes of roughly 2,000-10,000 km along a given field line, corresponding to emission at frequencies 100-800 kHz. This higher frequency emission is much less transient than that of higher altitude sources that emit between $\sim 30-100 \mathrm{kHz}$ and are well correlated with substorm onset (Morioka et al., 2007). To characterize the AKR observed by Wind and facilitate the comparison between the two spacecraft, the selected flux data are integrated over the frequency range 100-650 kHz. While the frequency range used by Lamy et al. (2010) (30-650 kHz) encompasses both the lower frequency and main band of AKR, we increase the lower frequency limit here to mitigate the inclusion of spurious data as mentioned above. While this does not allow for a direct comparison between 

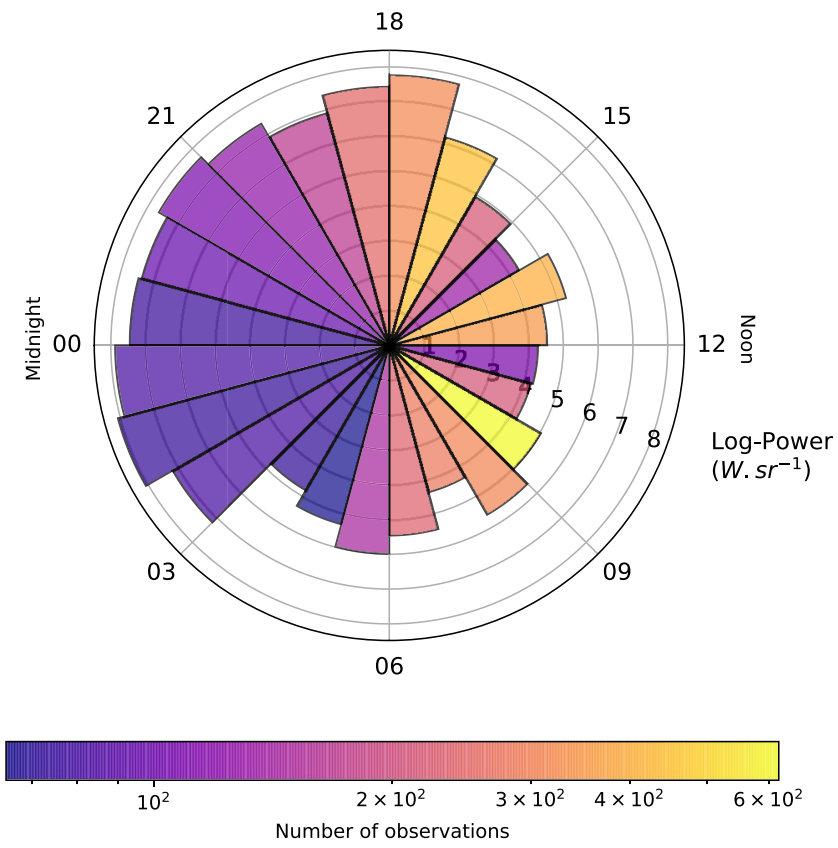

Figure 7. Log power in $\mathrm{Wsr}^{-1}$ integrated across the range $100-650 \mathrm{kHz}$ for the Auroral Kilometric Radiation (AKR) flux (as selected by 2.3) and averaged in local time (LT) bins $1 \mathrm{hr}$ wide. The sun and noon sector is to the right, while the midnight sector is to the left. Colors show the number of 3-minute-resolution observations, retained by the empirical selection in each LT bin. the integrated powers of Wind and Cassini, it enables general characteristics of the AKR, such as the viewing geometry and temporal modulations, to be studied. With a more refined selection of AKR signal at frequencies below $100 \mathrm{kHz}$, the power can be integrated over a frequency range such as $30-100 \mathrm{kHz}$ to investigate the lower frequency AKR component. Given that the integration time of each flux density measurement is the spin period of the spacecraft, the power can be integrated by simply taking the mean of the flux densities and integrating over the frequency channels. The flux densities have been normalized to $1 \mathrm{AU}$, so this distance is used as the effective area for the integration. Although we derive the AKR flux as that from a point source, the AKR is emitted in a wide geometry and so we present the AKR power as a fraction of the true beaming in units of $W s r^{-1}$.

\subsection{AKR Viewing Geometry}

Figure 7 shows the integrated power data described in the previous Section 3.2 after taking the mean power in $1 \mathrm{hr}$ spacecraft LT (GSE) bins and plotting the $\log _{10}$ power as a rose plot; the noon and midnight sectors are on the right and left of the figure, respectively, and the dawn and dusk sectors are at the bottom and on the top of the figure, respectively. A large asymmetry can be seen in the selected power with a 3-4 order of magnitude increase of the LT bins between 1700 and 0300 over those on the dayside that have the lowest powers, namely the LT sector at $1100 \mathrm{hr}$. The broad picture of Figure 7 is thus consistent with previous findings, which suggest that the source regions are located on the nightside and beam anisotropically (Alexander \& Kaiser, 1976; Gallagher \& Gurnett, 1979; Mutel et al., 2004, 2008). Also of interest here are the bins at 2100 and $2200 \mathrm{hr}$ LT, in the center of the range of intense emission. This corresponds to previous observations of the LT of the source regions most favored by AKR sources (Mutel et al., 2004; Panchenko, 2003), as well as the average LT of the most intense AKR source region at 2200 MLT (Green, 2004). Given the illumination region of an AKR source, and that the observations here are made from comparatively large radial distances and with a swept frequency receiver, it is expected that the emission from an AKR source could be measured by Wind from a neighboring LT sector. At Saturn, this longitudinal difference has been observed to be up to $2 \mathrm{hr}$ LT (Kimura et al., 2013; Lamy, Zarka, Cecconi, Prangé, et al., 2008). The location of the centroid of the most intense average power in Figure 7 is an indication of the effectiveness of the AKR selection used here.

While AKR has been previously observed from the dayside, it can be difficult, without polarization information, to discern whether this emission is attributable to the illumination of the spacecraft by a source on the nightside via either emission viewing geometry or scattering or that of a dayside source (Hanasz et al., 2003; Mutel et al., 2004; Schreiber et al., 2017). Deducing the exact origin of the source of emission is complex and is not possible within the scope of this study. However, the dynamic spectrogram in Figure 5 as well as the distribution of average power in Figure 7 show that we can observe AKR at all LT, with some of the most intense observations on the dayside made when Wind was within the LT sector 1300-1400 hrs and near the ecliptic plane.

The color of each LT bin reflects the number of data used to compute the average, where each data point represents the power of each 3- min frequency sweep. The orbital dynamics of the spacecraft produce the overall color distribution, with perigee on the nightside limiting the number of observations that can be made and vice versa on the dayside. For this reason, more total observations will be made on the dayside, but fewer of those observations will be selected given the preferred nightside location of the AKR source regions. This is seen when taking the ratio of the number of observations made in a local time sector with the number of selected data from the same sector. For the observations made on the dayside $(0600 \leq \mathrm{LT}$ $<1800)$, an average of $38 \%$ of data is retained by the selection, while $83 \%$ of nightside observations $(1800 \leq$ 

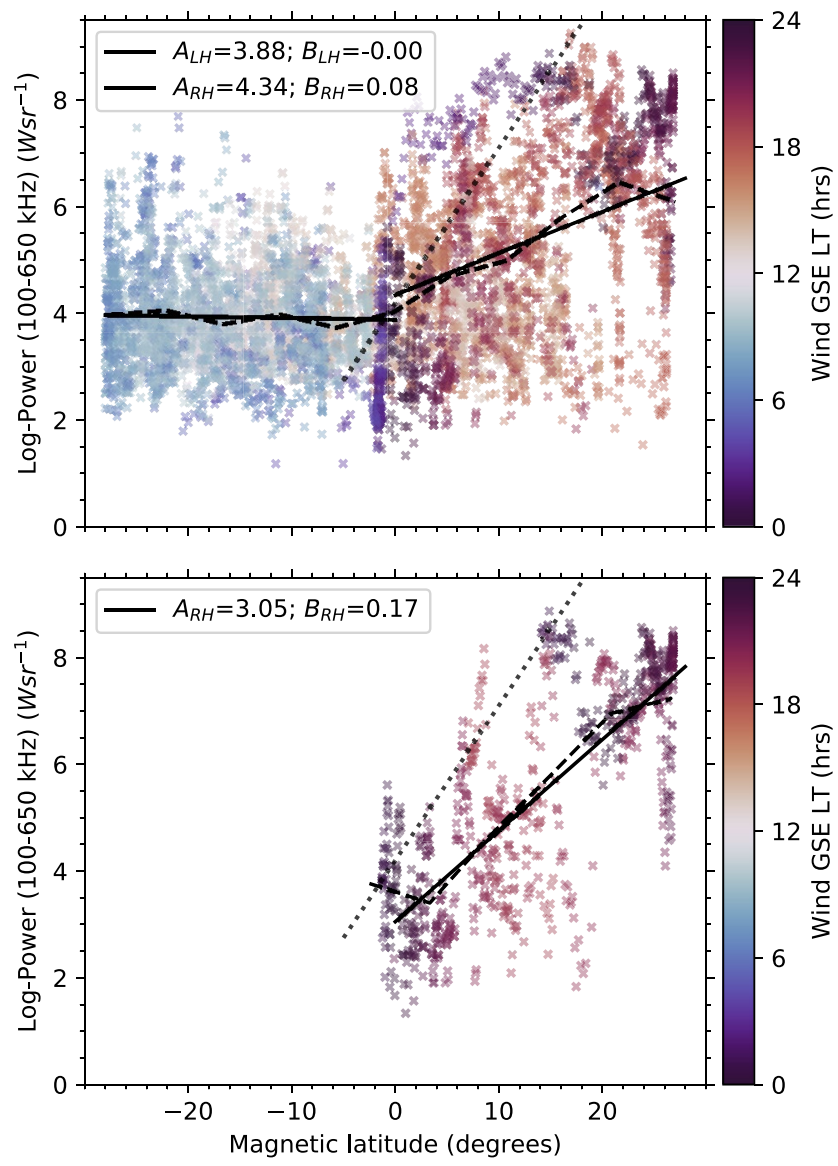

Figure 8. Distribution of Auroral Kilometric Radiation (AKR) power $(100-650 \mathrm{kHz})$ with magnetic latitude. The color bar shows the local time (LT) of Wind in geocentric-solar-ecliptic (GSE) coordinates, with observations in the Northern magnetic hemisphere made from the dusk flank, prior to perigee, and those in the Southern hemisphere from the dawn sector. The top panel shows all selected observations from day of year (DOY) 227-257, whilst the bottom panel shows a subset of observations made between 1900 and 0100 LT to account for poor viewing. The dashed black line shows the mean of the log-power after binning in $5^{\circ}$ wide latitude bins. The solid black lines show linear-log fits to the average data for negative and positive magnetic latitudes, where we expect dominant emission from left-handed circularly polarized ( $\mathrm{LH}$ ) to righthanded circularly polarized (RH) AKR, respectively. The legend indicates the coefficients (in ascending rank order) of the linear-log fit of the form shown in Equation 4. The dotted black line in each panel shows the analytic form found for Cassini RH AKR observations (see Equation 4; A $\sim 4.2$, B $=0.29$ ) (Lamy et al., 2010).
$\mathrm{LT}<0600$ ) are retained by the selection. The variation in the number of selected data in the noon sector in Figure 7 shows that the distribution here may not be indicative of the true average AKR power, as temporal variability of the state of the magnetosphere system will bias this count as the spacecraft crosses the dayside once during the 30- day period studied here. As discussed in Section 3.1, Wind crosses the nightside twice during this period, so the data contributing to the average on the nightside are comprised of two separate samples of this region. Further discussion of the power distributions that produced the averages for each LT bin can be found in Appendix D.

In addition to the viewing constraints introduced by the interaction of the terrestrial magnetosphere with the solar wind in Figure 7, the latitudinal distribution of AKR sources is such that we expect better viewing of AKR from individual magnetic hemispheres at higher latitudes. Figure 8 shows the distribution of AKR power with magnetic latitude with the LT of Wind indicated; while Wind was at relatively low magnetic latitudes for the period, it approached the nightside from the dusk flank at increasingly higher magnetic latitudes in the Northern magnetic hemisphere before crossing the magnetic equator and traveling to mid-latitudes as it leaves via the dusk flank. This can be seen in the top panel of Figure 8, which shows all selected data for the period. While powers were often observed as low as $10^{2} \mathrm{Wsr}^{-1}$ for all LT, the azimuthal viewing constraints of AKR are also seen as the average AKR power in the Southern magnetic hemisphere is relatively constant, indicating that Wind is not best situated to observe the most intense LH emission as it travels to the dayside, as shown also in Figure 7. The bottom panel of Figure 8 shows a smaller subset of the selected AKR data and attempts to mitigate the effect of the LT viewing, with only observations made between 1900 and 0100 LT included. While this LT range is slightly more restrictive than suggested by the distribution in Figure 7, it is chosen for a better characterization of the AKR for these observations. In both plots, the mean of the log-power observations made in $5^{\circ}$ wide magnetic latitude bins is shown, with a linear-logarithmic fit of the form used in equation 1 of Lamy et al. (2010), repeated here

$$
\log P=A+B \lambda_{W, m}
$$

where $P$ is the AKR power for a given hemisphere (e.g., LH or RH emission) in $\mathrm{Wsr}^{-1}$, and $\lambda_{W, m}$ is the magnetic latitude of Wind in degrees. Lamy et al. (2010) found, using the polarization to characterize each hemisphere, that the average emission from the Northern magnetic hemisphere, when integrated over 15 minutes, gave values $\mathrm{A} \sim 4.2, \mathrm{~B}=0.29$. Close to the magnetic equator, it is expected that AKR from both hemispheres will be observed, but their observations also showed that $\mathrm{RH}$ emission can be observed at $<10^{2} \mathrm{Wsr}^{-1}$ from magnetic latitudes of up to $5^{\circ}$ in the opposite hemisphere of origin (Southern). Without the polarization state we cannot discern the exact origin of emission, so the fit in Equation 4 is performed on data subset by negative or positive magnetic latitude, corresponding to assumed LH and RH AKR emission, respectively. The observations in the Southern hemisphere, made mostly on the dawn flank, show little dependence with latitude for reasons previously discussed. The differences between the positions of the spacecraft and the LT of Wind also clearly affect the observations made at positive magnetic latitudes, seen by the difference between the fit to positive latitudes in the two panels of Figure 8. The bottom panel shows better agreement with the RH power dependence observed by Cassini, with a shallower increase of the average power with latitude. In addition to Cassini being at a much greater distance from Earth and therefore, observing less low power AKR bursts, poorer temporal and spatial sampling from ideal viewing positions by 

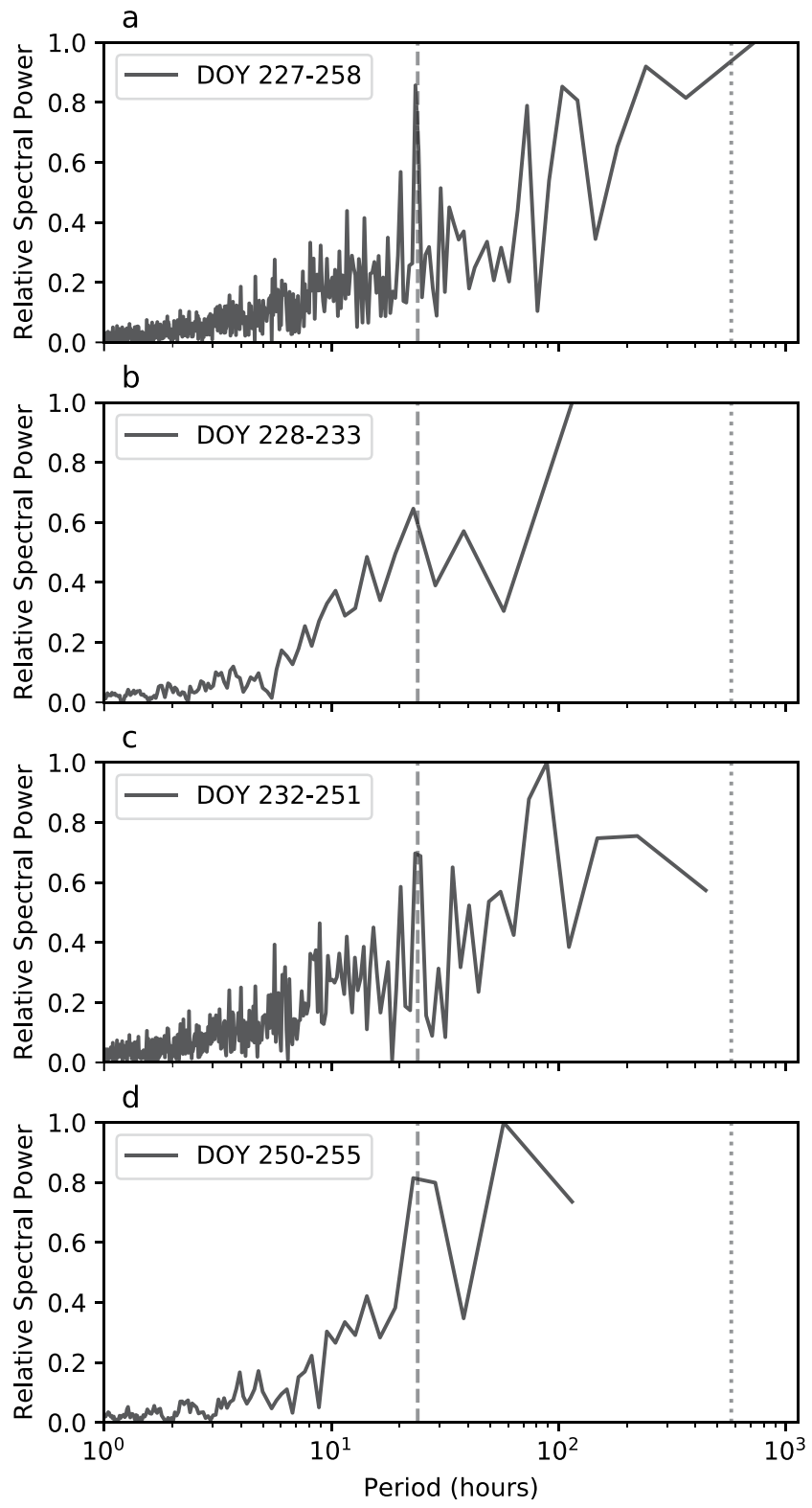

Figure 9. Fast Fourier Transforms (FFT) of the Auroral Kilometric Radiation (AKR) power, integrated over $100-650 \mathrm{kHz}$, for the whole 30- day period (a), a 5- day period that spans Wind's first nightside perigee (b), a 19- day period spanning Wind's apogee on the dayside (c) and a 5- day period spanning Wind's second nightside perigee (d). The legends in each panel show the 1999 day of year (DOY) for each subset, exclusive of the last date in each case. The integrated power is input at a 3- min resolution; data where no AKR is present are set to $P=10^{-8} \mathrm{Wsr}^{-1}$ to include them in the analysis. Analysis is performed on the integrated powers after applying a 3 - $\mathrm{hr}$ rolling window and log-transforming the data. The relative spectral power is shown; data of each panel are normalized by the value at the respective peak. The vertical gray dotted line is at a period of 24 days, the approximate orbital period of Wind, highlighting the peak period of Panel (a). The vertical gray dashed line shows a period of $24 \mathrm{hr}$.
Wind are assumed to produce the observed discrepancy. A full statistical characterization of the latitudinal dependence is out of the scope of this study and is not included.

\subsection{AKR Temporal Modulation}

To quantify the temporal variability of the AKR whilst accounting for the viewing, we perform a Fourier analysis on the entire time series as well as three subsets that comprise the two nightside passes/perigees of Wind during DOY 228-233 and 250-255, defined by a five-day period approximately centered on the midnight meridian and the dayside traversal during DOY 232-251. Figure 9 shows the result of a fast fourier transform (FFT) when applied to the Wind AKR power, integrated over $100-650 \mathrm{kHz}$, for the four aforementioned timeframes. The AKR power is initially averaged over a $3-\mathrm{hr}$ window to smooth the data and remove local temporal variability. Given that the integrated power can vary over several orders of magnitude, the FFT is performed after log-transforming the data to reduce the weight of this variability on the analysis. The relative spectral power is shown, having normalized the FFT output by the maximum spectral power found at the maximum period relevant to each timeframe. For example, the FFT output of the entire period in Figure $9 \mathrm{a}$ has the maximum peak at a period of 24 days $(576 \mathrm{hr})$, denoted by the vertical gray dotted line and corresponding to the approximate orbital period of Wind during this time. The presence of a peak at this period can be explained with reference to Figures $3 \mathrm{~b}$ and 5 ; the perigee of Wind precesses downward across the nightside magnetosphere, measuring intensifications in the AKR as it passes the nightside across two to three days, separated by the dayside apogee. We do not comment on the origin of the maximum peaks of the FFT in Figures 9b-9d.

The vertical dashed gray line in each panel of Figure 9 indicates a period of $24 \mathrm{hr}$. For Figure 9a, covering the entire 30- day period, the FFT output is noisy, particularly at lower frequencies but also at periods between 1 and 24 days. A peak of comparable power to the maximum at a 24- day period is seen at a period of $24 \mathrm{hr}$, showing the observation of the diurnal modulation of AKR. Figures $9 \mathrm{~b}$ and $9 \mathrm{~d}$, including data corresponding to the two nightside perigees, also both show peaks at 24- hr periods, with the spectral resolution being limited by the length of these time series. Figure 9c, corresponding to apogee on the dayside, also shows a diurnal modulation; each period was tested for statistical significance with bootstrapping, where the time series is shuffled randomly before undergoing the same analysis and comparing the spectral peak, allowing the null hypothesis to be tested. We found that the peak at $24 \mathrm{hr}$ for each panel in Figure 9 has a $p$-value of $10^{-5}$ and so is significant. While the presence of diurnal modulation has been previously observed and is a validation of the empirical selection used here in itself, a significant peak in Wind observations on the dayside further corroborates the hypothesis that the diurnal modulation is predominantly due to a geometric viewing effect. Given that the diurnal modulation is seen during observations near noon, and near the magnetic equator, we expect that intrinsic variability of the AKR sources is unobservable and so such a modulation is likely due to the extremes of the emission cones illuminating Wind as the magnetic dipole tilts. 

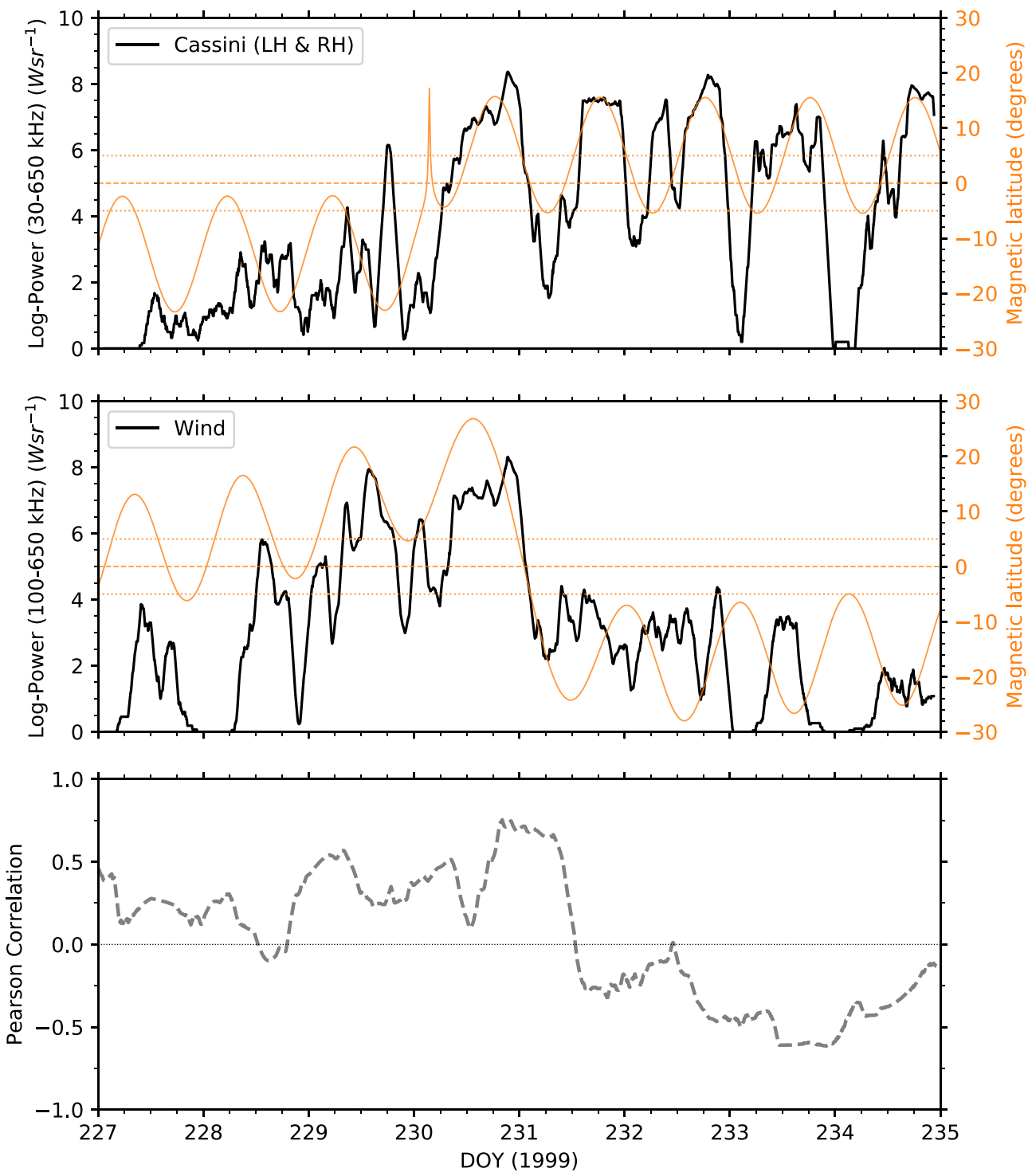

Figure 10. Comparative time series of Auroral Kilometric Radiation (AKR) power for the eight days (day of year [DOY] 227-235) that comprise Wind's first perigee, taking it across the nightside. The top panel shows the Cassini integrated power (30-650 kHz) averaged over a moving 3- hr window and including both left-handed circularly polarized (LH) and left-handed circularly polarized (RH) emission. The middle panel shows the 3-hour-averaged Wind integrated power (100-650 kHz). Shown in orange on the right ordinate of both the top and middle panels is the magnetic latitude of the respective spacecraft. Also shown in the top and middle panels are lines indicating the magnetic equator and $\pm 5^{\circ}$. The bottom panel shows the linear cross-correlation between the AKR power as observed by Wind and the magnetic latitude of the spacecraft, with a 24- hr moving window.

For a highly directional emission such as AKR, with spatially complex source regions and triggering via coupling to the dynamic magnetosphere, we can examine the temporal variability observed from each spacecraft's position to attempt to further explain these diurnal modulations. Figure 10 shows time series of the AKR power observed by Wind and Cassini for DOY 227-235, during which Wind traveled across the nightside from the dusk to dawn sectors, crossing the magnetic equator around DOY 231. The power shown for Cassini in the top panel is for both LH and RH AKR, is integrated over the range $30-650 \mathrm{kHz}$, and is averaged similarly over a 3- $\mathrm{h}$ window. $3 \mathrm{~h}$ averages of the Wind AKR power are shown in the middle panel, but again integrated over the range $100-650 \mathrm{kHz}$ for reasons given in Section 3.2. Although we expect some discrepancy in the magnitude of the average power for this reason, the effects of this will be negligible compared to those introduced by the viewing positions and it is preferable to compare this range to reduce 
the contamination from low frequencies. Given the dependence of viewing of the AKR from a particular hemisphere on the magnetic latitude as observed by Cassini and shown in figure 8 of Lamy et al. (2010), we can assume that the dominant AKR observed by a spacecraft at a magnetic latitude $\left|\lambda_{m}\right|>5^{\circ}$ will be of the corresponding magnetic hemisphere, with little contribution from the other hemisphere if observed. Visual inspection of the AKR power from both spacecraft shows that the bursts are not only seen to be antiphased when in opposite hemispheres, but that they can also be phased on various timescales regardless of the spacecraft position. A particularly strong example of this is seen in the peak $\sim 10^{3} \mathrm{Wsr}^{-1}$ in the AKR power observed by Wind when between $-20^{\circ}$ and $-30^{\circ}$ magnetic latitude, centered on DOY 233.5, whilst Cassini sees a peak of $\sim 10^{7} \mathrm{Wsr}^{-1}$ whilst close to the magnetic equator at positive latitudes.

Given the significant diurnal modulation, we can attempt to discern its origin by examining the correlation of the AKR bursting with the magnetic latitude of the observing spacecraft, here Wind. The bottom panel of Figure 10 shows the linear cross-correlation of the AKR power with the magnetic latitude over a 24- $\mathrm{hr}$ moving window. While a similar analysis is performed over a 5- day window in Lamy et al. (2010), the data coverage of Wind while in an appropriate viewing position is insufficient for this. While the choice of the window size is arbitrary, this was chosen to examine correlation features on a similar timescale to the modulation in question. While the correlation fluctuates, the AKR power is mostly correlated positively with the magnetic latitude of Wind, with the correlation increasing as Wind travels to higher latitudes in the Northern magnetic hemisphere, away from the region in which the emission from either hemisphere is observable and of a comparable intensity (cf., figure 8 of Lamy et al. (2010)). Once wind crosses the magnetic equator at the start of DOY 231, the AKR power becomes anti-correlated with Wind's magnetic latitude after perigee; whilst the AKR power decreases as Wind travels away from the nightside and into the dawn sector, the time series clearly shows the dominant emission from the Southern magnetic hemisphere after Wind crosses the magnetic equator. These observations are consistent with those of Cassini, which showed an anti-correlated diurnal modulation of the RH and LH AKR power, with each correlating with the magnetic latitude of the corresponding magnetic hemisphere. This was interpreted as a result of the diurnal rocking of the AKR source region and the beaming pattern, and this is assumed to be the dominant source of the modulation observed here. On the other hand, the presence of phased bursts in observations of both spacecraft regardless of magnetic latitude is contrary to this. While AKR observations with Wind alone appear to be consistent with those of Cassini, with peak AKR power observed at extremes of the spacecraft magnetic latitude and suggesting dominance of a viewing effect, the phasing between AKR bursts observed by both spacecraft brings another new and important constraint to the origin of the diurnal modulation. While the combination of the Wind and Cassini observations here has again illustrated the value of multipoint measurements of AKR, the detailed investigation of the burst phasing is beyond the scope of this study.

\section{Conclusion}

We have described a new method of selecting AKR emission from the complex radio environment observed by Wind, using a statistical measure of the variability of radio flux across the spin-axis-aligned $\mathrm{Z}$ antenna during a spacecraft spin. Examination of individual spins and the flux density dynamic spectrograms during a 30- day trajectory of Wind shows that the selection is effective at removing ubiquitous solar Type III bursts from the data and isolating AKR (Figures 1 and 2). Although there are limitations at lower frequencies as RFI and sources of high temporal variability from the local plasma can contaminate the selection, the selection criterion employed here is based on a simple numeric threshold and can be readily applied to the extensive data set of Wind. Here, we applied the AKR selection to Wind data for an interval overlapping with the Earth flyby of the Cassini spacecraft. The Cassini data have previously been treated with a full GP inversion (Lamy et al., 2010), but here they provide context for the sensitivity of radio observations to the viewing position of the spacecraft. After considering the discrepancies between the viewing positions of the two spacecraft during the period (Figures 3 and 4), the flux density dynamic spectrograms of the 30- day period between Wind and Cassini are compared in Figure 5. This shows the expected reduction in observed emission as Wind traverses the dayside and is no longer illuminated by the most intense AKR sources located on the nightside. Examining the data more closely, we compare the flux density spectra of Wind observations made from a comparable region to that of Cassini for the period and find that the general characteristics of the AKR spectrum are reproduced well at frequencies ) $E \mathrm{kHz}$. Figure 6 shows the broadening 
of the spectral peak at around $200 \mathrm{kHz}$ in the median spectrum to higher frequencies for the most intense emission, agreeing with the accepted AKR spectrum and the previous Cassini observations.

Accounting for the aforementioned limitations, we integrate the flux densities measured by Wind between 100 and $650 \mathrm{kHz}$ for each 3- min sweep to represent the confident selection of AKR, covering the main frequency range and allowing a comparison with previous results from Cassini. We have accounted for the viewing geometry of AKR in the observations by averaging the integrated power measured in each onehour LT bin shown in Figure 7. This reproduces quantitatively the day-night asymmetry that can be seen in Figure 5 and is again expected, with a 4 order of magnitude increase from $\sim 10^{4}-10^{8} \mathrm{Wsr}^{-1}$. This also provides confidence in the selection of AKR by this method, given that it also reproduces the dawn-dusk asymmetry associated with the close correlation between the AKR source region and the auroral region in the ionosphere. Emission is selected at all LTs, showing that we can observe AKR from many of Wind's various viewing positions in the magnetosphere and solar wind and shows promise for future studies where the selection can be applied to a larger Wind data set and aid statistical analyses of events. We also examine in Figure 8 the dependence of the average AKR power with the magnetic latitude of the observation. While we cannot confirm a latitudinal dependence of the AKR power for sources observed from the dayside, Wind observations made at ideal LT show general agreement with the relationship found for RH AKR observed by Cassini in the Northern magnetic hemisphere.

Using the selected integrated power, we examined the temporal variability of the Wind AKR observations, confirming the presence of a diurnal modulation with an FFT analysis shown in Figure 9. We observe a statistically significant $\left(p<10^{-5}\right)$ peak at $24 \mathrm{hr}$ for the entire period, as well as for three subsets of the data corresponding to the first perigee from DOY 228-233, the traversal of the dayside from DOY 232-251 and the second perigee from DOY 250 to 255. In Figure 10, we compared in more detail the AKR power between Wind and Cassini during the first perigee of Wind, which placed it ideally to observe intrinsic AKR modulation over a few days. A linear cross-correlation between the Wind AKR power and the magnetic latitude of the spacecraft showed that the AKR is generally correlated in the Northern magnetic hemisphere and then anti-correlated as it crosses into the Southern, lending weight to the visibility of the AKR emission cone in each hemisphere as the predominant source of the diurnal modulation. On the contrary, however, AKR bursts are also observed that are in phase whilst the spacecraft are in separate magnetic hemispheres, although only a short time period is shown here and further inference is out of the scope of the study. Given the ease of masking the Wind data with this technique, longer-term studies of diurnal and semi-diurnal modulations can be conducted with Wind alone, which has $>2$ decades of observations from a variety of positions. With the verification of the empirical selection as seen here, statistical analyses can also be conducted between resulting AKR data set and lists of substorm onsets that cover decades and are complimentary to Wind's lifetime.

\section{Appendix A: Errors Due to Assumption of Source Direction}

As described in Section 2.2.2, we do not have an exact indicator of the AKR emission hemisphere and expect that both LH and RH AKR will illuminate Wind during certain times is dependent on its viewing position. AKR source regions are known to be confined to nightside LT along high latitude magnetic field lines, which allow the geographic or magnetic poles to be used as a reasonable assumption for the source center. While an appropriate pole could be chosen based on the sign of the spacecraft magnetic latitude, for example, it is favourable to apply a consistent assumption to all observations for this general selection algorithm. Figure A1 shows the distribution of errors introduced by using the Earth's center over the poles. $\lambda_{P}$, the apparent latitude of Wind when translating the ecliptic plane to either geographic pole, gives a proxy of the geographic pole direction from Wind. From Equation 2, we compare the ratio $\frac{\cos ^{2} \lambda_{P}}{\cos ^{2} \lambda_{G S E}}$ to characterize the implicit error introduced here and find that the maximum value in the distribution is $1.2 \%$. 


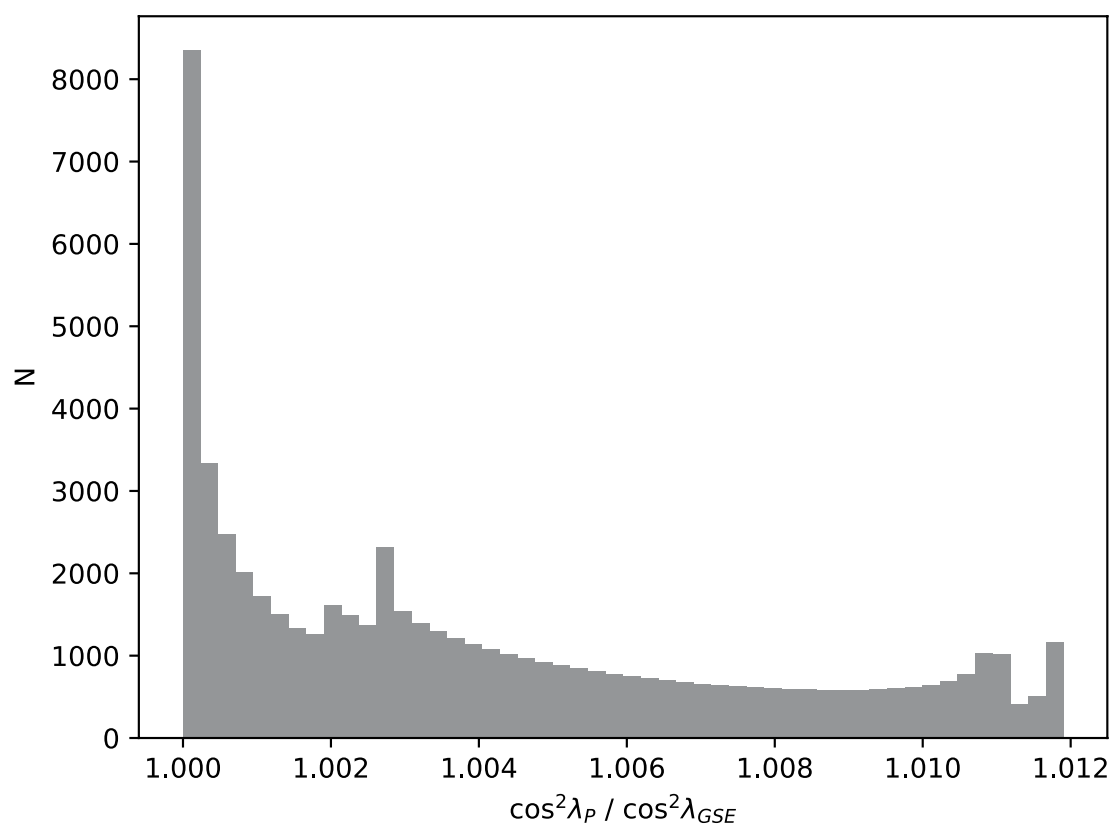

Figure A1. Statistical characterization of the error implicit in the assumption of Earth's center as the direction of the Auroral Kilometric Radiation (AKR) source. Presented data relates only to the 30- day period studied here and shows the fraction of Wind observations that would be affected by a given error factor. $\cos ^{2} \lambda_{G S E}$ is the term used in Equation 2, while $\cos ^{2} \lambda_{P}$ is the term calculated using the apparent latitude of Wind with respect to geographic poles.

\section{Appendix B: Cross-Checking Flux Densities Using Type III Bursts}

As mentioned in Section 2.2.2, we initially compared the flux density resulting from Equation 2, derived from the linear $\mathrm{Z}$ antenna, with those from a full GP inversion. Although the scaling factor that results from this work is not applied, we retain the results here as a point of interest, given the observed discrepancies. We have access to results from a GP inversion that assumes the source parameters of a solar radio Type III burst (namely that they are unpolarized) and utilizes the Wind/WAVES system such that the total flux density is retrieved. The $S$ ' antenna, used explicitly to retrieve the degree of circular polarization, is thus ignored in this inversion. To compare the fluxes from the $\mathrm{Z}$ antenna to GP fluxes, we must omit the $\cos ^{2} \lambda$ term from Equation 2 to reflect the fact that the source direction is no longer assumed to be at Earth, and the Type III burst source region is sufficiently far enough away and close to the ecliptic plane that the $\mathrm{Z}$ antenna is always perpendicular to the emission. Explicitly, the $\mathrm{Z}$ antenna is pre-calibrated using

$$
P_{Z}=\frac{1}{2} G S_{Z}
$$

for the observations presented in this section. The fluxes from the Type III inversion with Wind are derived using a calibration from Zarka et al. (2004), which has since been corrected by a factor 2 by Zaslavsky et al. (2011) and is accounted for here. The fluxes from the Type III inversion with Cassini are similarly derived but have already accounted for this factor (Cecconi et al., 2017). 

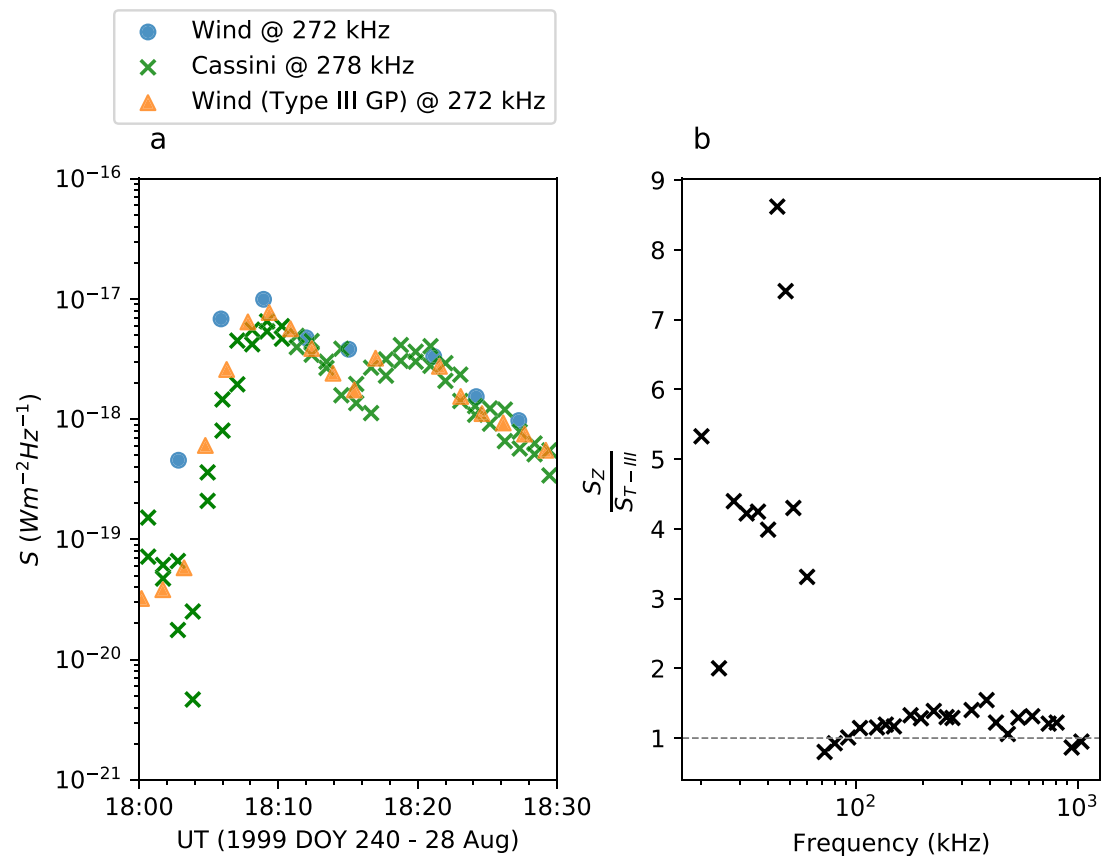

Figure B1. Comparing flux densities of a single Type III burst at comparable frequency channels between spacecraft (left) and the resulting ratio between the two datasets from Wind, using the peak flux spectra from the Type III burst (right). Included in the left panel are data derived from Wind using the calibration method described in Section 2.2.2 ( $S_{Z}$, blue circles), modified to account for the change in radio source (see Appendix B), data from Cassini using a GP inversion that treats Type III bursts (green crosses) and data from Wind using a similarly modified GP inversion at the original $90 \mathrm{~s}$ resolution of the frequency channel $\left(S_{T-I I I}\right)$, orange triangles. The panel on the right shows the ratio $\frac{S_{Z}}{S_{T-I I I}}$, where each data point is given by the ratio of the peak flux between data sets during DOY 240 18:00-18:30. The gray, horizontal dashed line is at unity.

Figure B1 shows the comparison of calibrated flux densities for an example of a Type III burst observed by Wind and Cassini during 1999 DOY 240. The Wind observations are given at different resolutions; the data used here are averaged over the 3- min sweep, while the original resolution of each frequency channel is retained in the data from the Type III GP inversion with Wind. The peak of the primary Type III burst at $272 \mathrm{kHz}$ is seen close to 18:10 UT in this example, and while good agreement is seen between the Type III GP inversions with Cassini and Wind, the $\mathrm{Z}$ antenna-calibrated data have an approximately constant offset following the peak. By defining the bounding UT of the Type III burst as well as its frequency limits following visual examination of the relevant dynamic spectrogram, we can track the peak flux of the Type III burst for each set of fluxes and produce a spectrum of the ratio $\frac{S_{Z}}{S_{T-I I I}}$, where $S_{Z}$ is the flux from Equation B1 and $S_{T-I I I}$ is the flux from the Type III GP inversion with Wind. The example in Figure B1 shows the entire Wind/WAVES RAD1 spectrum, including the lower frequencies that the Type III emission does not reach and showing the discrepancy between the two data below $\sim 70 \mathrm{kHz}$. While we do not explore it in detail, it is interesting to note that the Cassini observations, derived with an inversion adapted to AKR sources, sees good agreement with the Wind observations from the Type III inversion. 


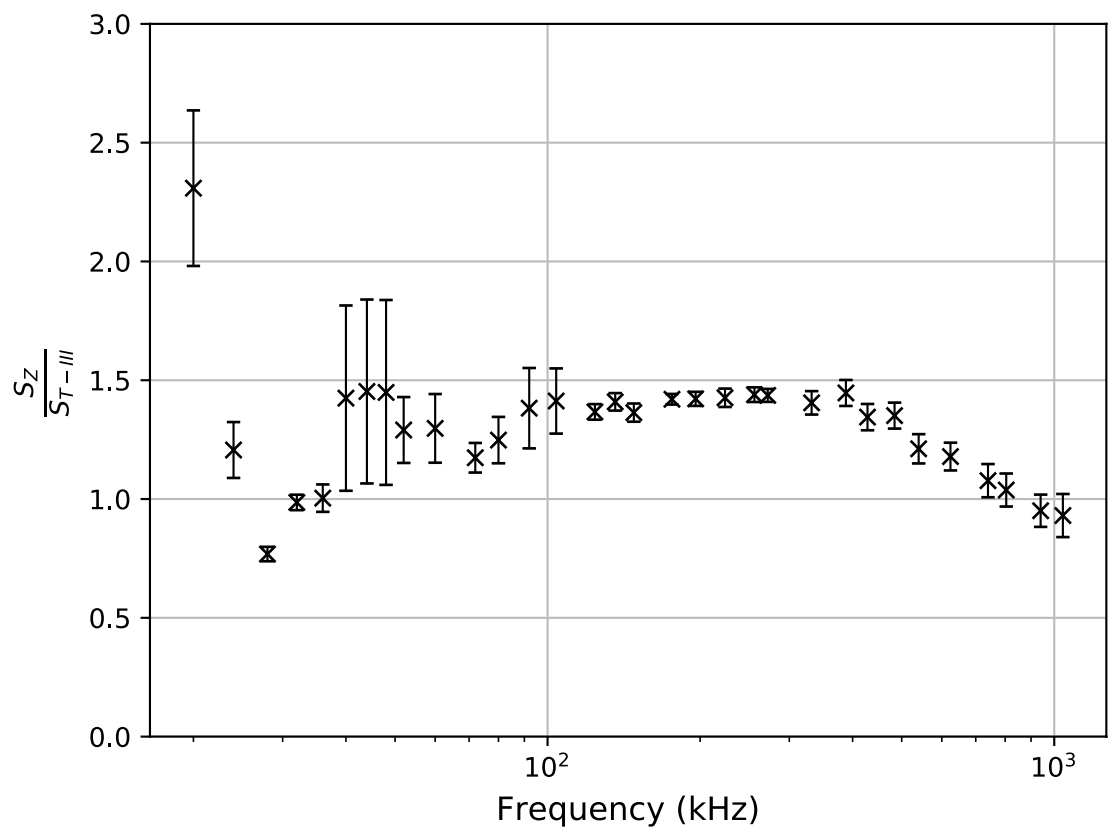

Figure B2. Spectrum of the average ratio between peak flux spectra for a total of 19 Type III bursts. As detailed in section Appendix B, each Type III example is described by a start and end time as well as its frequency limits. A spectrum is formed for each example by taking the ratio of the peak fluxes in each channel between the bounding times (see text). The average spectrum shows the mean of the ratios of each frequency channel, with error bars showing the standard error of the mean.

Figure B2 shows the mean $\frac{S_{Z}}{S_{T-I I I}}$ spectrum from a set of 19 Type III bursts. The uncertainties on the cross-calibration spectrum are given by the standard error of the mean and show the better-constrained values at frequencies $>50 \mathrm{kHz}$ for which the majority of the selected Type III bursts are emitting. $\frac{S_{Z}}{S_{T-I I I}}$ tends to increase at the lowest frequencies, with the most pronounced increase seen at $20 \mathrm{kHz}$. This is likely due to the inclusion of spurious emission from the visual examination, as well as the limited number of Type III bursts (7) that were emitting at this frequency. The fact that the linear $\mathrm{Z}$ antenna observes calibrated fluxes larger than those from two independent sets of observations using GP inversions with Wind and Cassini is puzzling, but we do not explore the details for this here.

\section{Appendix C: $\sigma_{Z}$ Threshold Justification}

Figure $\mathrm{C} 1$ shows the distribution of $\sigma_{Z}$ values observed at $272 \mathrm{kHz}$ and during the 30- day period studied here. This frequency channel was chosen to present as it is a good representation of the typical peak frequency of the AKR spectrum. Shown in the plot is the value used to select AKR data as described in Section 2.3; at values lower than this the majority of the emission is found, with a strong Gaussian profile centered roughly on $10^{-1.5}$ representing the majority of other emission such as Type III bursts and background sources, while at values higher than $10^{-1}$, a second population is seen that contains AKR as well as low frequency contaminants. While determining the exact $\sigma_{Z}$ distribution of AKR observations for the entire spectral range is out of the scope of this study, examinations of this metric at other frequencies exhibit similar distributions that lend weight to this choice of the threshold. 


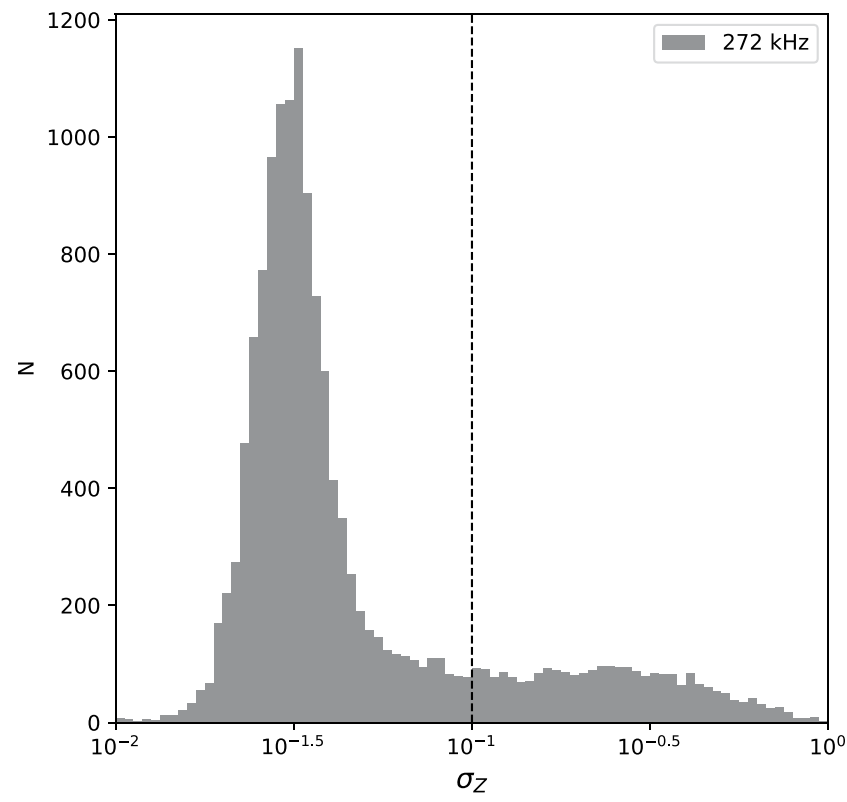

Figure C1. Distribution of $\sigma_{Z}$ values for all observations made at $272 \mathrm{kHz}$ during the 30 - day period studied here. The black, vertical dashed line shows the value of the threshold $\left(\sigma_{Z} \geq 0.1\right)$ chosen to select Auroral Kilometric Radiation (AKR) data.

\section{Appendix D: AKR Power Distributions With Spacecraft LT}

Figure D1 shows the distribution of integrated power observations (100-650 kHz) for each LT bin used to compute the average values for Figure 7. From the top and bottom rows, corresponding mostly to LT sectors that Wind traveled through on two, separate occasions, evidence can be seen that suggests that the distributions are comprised of two separate events of AKR emission which differ in intensity, whether due to intrinsic differences due to the current state of the magnetosphere or other effects. Some of the bins exhibit two distributions that are separated by 6 orders of magnitude in some cases (e.g., 01-02 LT), as well as non-Gaussian distributions (e.g., 08-09 LT). Applying the selection technique over a longer period will remove some of these local effects and better characterize the statistical AKR power distribution with LT. Taking the mean of the distributions here gives an average that is skewed toward higher values and represents more closely the larger extreme of the total distribution of data, as seen by its position with respect to the $75 \%$ quantile in most panels of Figure D1. The median of these distributions would be a more statistically rigorous measure of the average. Here, however, where the aim is to demonstrate the empirical selection of AKR data and not to rigorously define the average AKR power of these observations with LT, the mean is sufficient. 

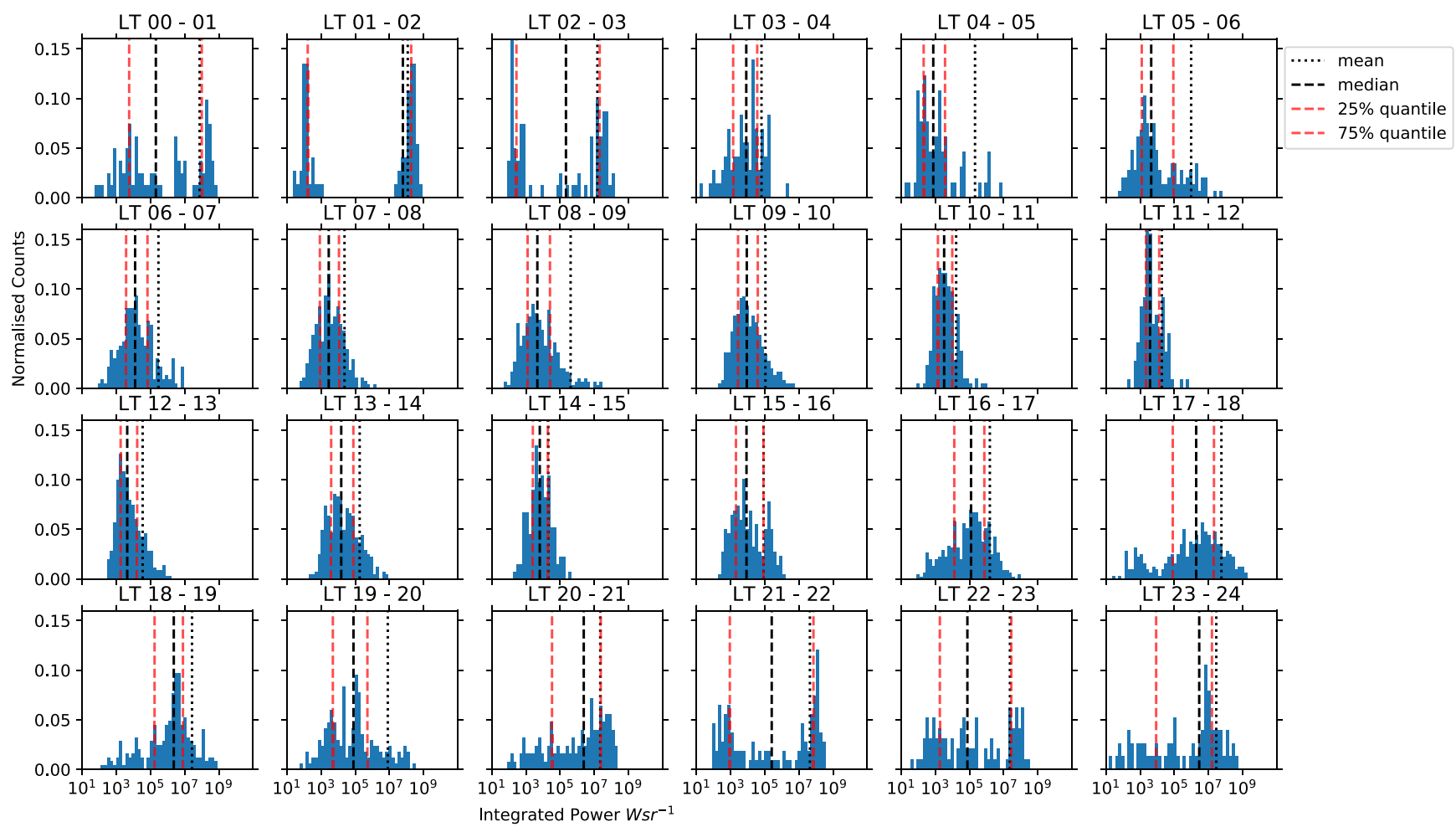

Integrated Power Wsr-1
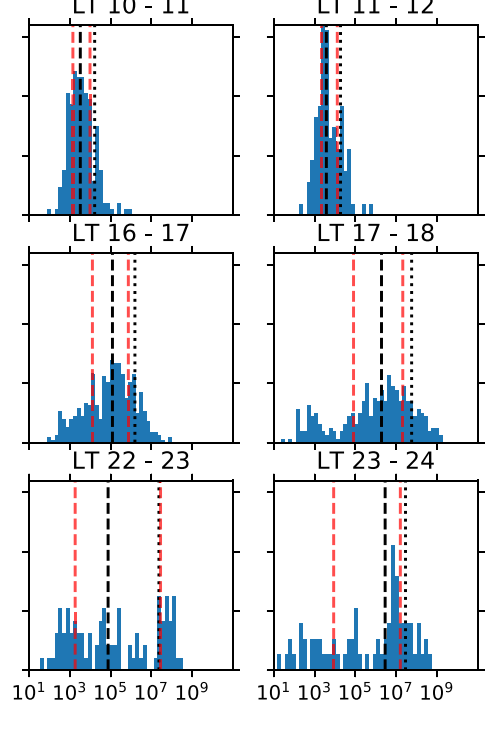

Figure D1. Normalized histograms showing the distribution of log integrated power in each $1 \mathrm{hr}$ local time (LT) bin used to create Figure 7. Each histogram has black dashed and red dashed vertical lines to represent the median and lower and upper quartiles of the distributions, while the black dotted line shows the mean.

Acknowledgments

J. E. Waters's work was supported by the EPSRC Centre for Doctoral Training in Next Generation Computational Modelling Grant No. EP/L015382/1. C. M. Jackman's work is supported by the Science Foundation Ireland Grant 18/ FRL/6199. D. K. Whiter was supported by the Natural Environment Research Council of the UK under grant NE/ S015167/1. The authors acknowledge CNES (Centre National d'Etudes Spatiales), CNRS (Centre National de la Recherche Scientifique), and Observatoire de Paris for support to the Wind/ Waves team and the CDPP (Centre de Données de la Physique des Plasmas) for the provision of the Wind/Waves RAD1 L2 data. The authors acknowledge support from Paris Astronomical Data Centre (PADC) for the preparation and distribution of the data collection.

\section{Data Availability Statement}

The Cassini RPWS data from the GP inversion during the period studied here are included in Lamy et al. (2010) and can be found online (https://doi.org/10.25935/5JFX-DH49 (Cecconi et al., 2017)). The data used in this study can be found online (https://doi.org/10.25935/wxv0-vr90), as can the code used to calibrate and apply the AKR selection (https://github.com/WatersJE/WindWaves_AKR_calibration_selection).

\section{References}

Akasofu, S. I. (1964). The development of the auroral substorm. Planetary and Space Science, 12(4), 273-282. https://doi. org/10.1016/0032-0633(64)90151-5

Alexander, J. K., \& Kaiser, M. L. (1976). Terrestrial kilometric radiation, 1. Spatial structure studies. Journal of Geophysical Research, 81(34), 5948-5956. https://doi.org/10.1029/ja081i034p05948

Anderson, R. R., Matsumoto, H., Hashimoto, K., Kojima, H., Kasaba, Y., Kaiser, M. L., et al. (2005). Geotail, polar, and wind observations of Auroral kilometric radiation. COSPAR Colloquia Series, 16(C), 205-219. https://doi.org/10.1016/S0964-2749(05)80032-0

Bougeret, J. L., Kaiser, M. L., Kellogg, P. J., Manning, R., Goetz, K., Monson, S. J., et al. (1995). Waves: The radio and plasma wave investigation on the wind spacecraft. Space Science Reviews, 71(1-4), 231-263. https://doi.org/10.1007/BF00751331

Cane, H. V. (1979). Spectra of the non-thermal radio radiation from the galactic polar regions. Monthly Notices of the Royal Astronomical Society, 189(3), 465-478. https://doi.org/10.1093/mnras/189.3.465

Cecconi, B. (2019). Polarization in low frequency radio astronomy (Vol. 1-26). Retrieved from http://arxiv.org/abs/1901.03599

Cecconi, B., Lamy, L., \& Zarka, P. (2017). Cassini/rpws/hfr lesia/kronos n3d data collection (version 1.0) [data set]. PADC. https://doi. org/10.25935/5JFX-DH49

Cecconi, B., \& Zarka, P. (2005). Direction finding and antenna calibration through analytical inversion of radio measurements performed using a system of two or three electric dipole antennas on a three-axis stabilized spacecraft. Radio Science, 40(3), 1-20. https://doi. org/10.1029/2004RS003070

Dulk, G. A., Erickson, W. C., Manning, R., \& Bougeret, J.-L. (2001). Calibration of low-frequency radio telescopes using the galactic background radiation. Astronomy and Astrophysics, 365(2), 294-300. https://doi.org/10.1051/0004-6361:20000006

Gallagher, D. L., \& Gurnett, D. A. (1979). Auroral kilometric radiation: Time-averaged source location. Journal of Geophysical Research, 84(A11), 6501-6509. https://doi.org/10.1029/JA084iA11p06501

Green, J. L. (2004). Seasonal and solar cycle dynamics of the auroral kilometric radiation source region. Journal of Geophysical Research, 109(A5), A05223. https://doi.org/10.1029/2003ja010311 
Green, J. L., Gurnett, D. A., \& Shawhan, S. D. (1977). The angular distribution of auroral kilometric radiation. Journal of Geophysical Research, 82(13), 1825-1838. https://doi.org/10.1029/ja082i013p01825

Gurnett, D. A. (1974). The Earth as a radio source: Terrestrial kilometric radiation. Journal of Geophysical Research, 79(28), 4227-4238. https://doi.org/10.1029/ja079i028p04227

Hanasz, J., Panchenko, M., De Feraudy, H., Schreiber, R., \& Mogilevsky, M. M. (2003). Occurrence distributions of the auroral kilometric radiation ordinary and extraordinary wave modes. Journal of Geophysical Research, 108(A11), 1408. https://doi.org/10.1029/2002JA009579

Hillan, D. S., Cairns, I. H., Robinson, P. A., \& Mohamed, A. (2010). Prediction of background levels for the wind waves instrument and implications for the galactic background radiation. Journal of Geophysical Research, 115(A6), 1-11. https://doi.org/10.1029/2009ja014714

Juusola, L., Østgaard, N., Tanskanen, E., Partamies, N., \& Snekvik, K. (2011). Earthward plasma sheet flows during substorm phases. Journal of Geophysical Research, 116(A10), A10228. https://doi.org/10.1029/2011JA016852

Kaiser, M. L., \& Alexander, J. K. (1977). Terrestrial kilometric radiation III: Average spectral properties. Journal of Geophysical Research, $22,82$.

Kaiser, M. L., Alexander, J. K., Riddle, A. C., Pearce, J. B., \& Warwick, J. W. (1978). Direct measurements by Voyagers 1 and 2 of the polarization of terrestrial kilometric radiation. Geophysical Research Letters, 5(10), 857-860. https://doi.org/10.1029/gl005i010p00857

Kasaba, Y., Matsumoto, H., Hashimoto, K., \& Anderson, R. R. (1997). The angular distribution of auroral kilometric radiation observed by the GEOTAIL spacecraft. Geophysical Research Letters, 24(20), 2483-2486. https://doi.org/10.1029/97gl02599

Kepko, L., McPherron, R. L., Amm, O., Apatenkov, S., Baumjohann, W., Birn, J., \& Sergeev, V. (2015). Substorm current wedge revisited. Space Science Reviews, 190(1-4), 1-46. https://doi.org/10.1007/s11214-014-0124-9

Kimura, T., Lamy, L., Tao, C., Badman, S. V., Kasahara, S., Cecconi, B., \& Fujimoto, M. (2013). Long-term modulations of Saturn's auroral radio emissions by the solar wind and seasonal variations controlled by the solar ultraviolet flux. Journal of Geophysical Research: Space Physics, 118(11), 7019-7035. https://doi.org/10.1002/2013JA018833

Krupar, V., Maksimovic, M., Kontar, E. P., Zaslavsky, A., Santolik, O., Soucek, J., \& Szabo, A. (2018). Interplanetary type III bursts and electron density fluctuations in the solar wind. The Astrophysical Journal, 857(2), 82. https://doi.org/10.3847/1538-4357/aab60f

Lamy, L., Zarka, P., Cecconi, B., Hess, S., \& Prangé, R. (2008). Modeling of Saturn kilometric radiation arcs and equatorial shadow zone. Journal of Geophysical Research, 113(10), 1-10. https://doi.org/10.1029/2008JA013464

Lamy, L., Zarka, P., Cecconi, B., \& Prangé, R. (2010). Auroral kilometric radiation diurnal, semidiurnal, and shorter-term modulations disentangled by Cassini. Journal of Geophysical Research, 115(A9), A09221. https://doi.org/10.1029/2010ja015434

Lamy, L., Zarka, P., Cecconi, B., Prangé, R., Kurth, W. S., \& Gurnett, D. A. (2008). Saturn kilometric radiation: Average and statistical properties. Journal of Geophysical Research, 113(A7), A07201. https://doi.org/10.1029/2007ja012900

Liou, K. (2002). Magnetic dipolarization with substorm expansion onset. Journal of Geophysical Research, 107(A7), 1131. https://doi. org/10.1029/2001ja000179

Liou, K., Meng, C.-I., Lui, A. T. Y., Newell, P. T., \& Anderson, R. R. (2000). Auroral kilometric radiation at substorm onset. Journal of Geophysical Research, 105(A11), 25325-25331. https://doi.org/10.1029/2000ja000038

Manning, R., \& Fainberg, J. (1980). A new method of measuring radio source parameters of a partially polarized distributed source from spacecraft observations. Space Science Instrumentation, 5, 161-181.

McPherron, R. L., Russell, C. T., \& Aubry, M. P. (1973). Satellite studies of magnetospheric substorms on August 15, 1968: 9. Phenomenological model for substorms. Journal of Geophysical Research, 78(16), 3131-3149. https://doi.org/10.1029/ja078i016p03131

Menietti, J. D., Mutel, R. L., Christopher, I. W., Hutchinson, K. A., \& Sigwarth, J. B. (2011). Simultaneous radio and optical observations of auroral structures: Implications for AKR beaming. Journal of Geophysical Research, 116(12), 1-9. https://doi.org/10.1029/2011JA017168

Meyer-Vernet, N., Hoang, S., Issautier, K., Maksimovic, M., Manning, R., Moncuquet, M., \& Stone, R. (1998). Measuring plasma parameters with thermal noise spectroscopy. Measurement Techniques in Space Plasmas.

Meyer-Vernet, N., Issautier, K., \& Moncuquet, M. (2017). Quasi-thermal noise spectroscopy: The art and the practice. Journal of Geophysical Research: Space Physics, 122(8), 7925-7945. https://doi.org/10.1002/2017JA024449

Meyer-Vernet, N., \& Perche, C. (1989). Tool kit for antennae and thermal noise near the plasma frequency. Journal of Geophysical Research, 94(A3), 2405. https://doi.org/10.1029/ja094ia03p02405

Morioka, A., Miyoshi, Y., Kurita, S., Kasaba, Y., Angelopoulos, V., Misawa, H., \& McFadden, J. P. (2013). Universal time control of AKR: Earth is a spin-modulated variable radio source. Journal of Geophysical Research: Space Physics, 118(3), 1123-1131. https://doi. org/10.1002/jgra.50180

Morioka, A., Miyoshi, Y., Tsuchiya, F., Misawa, H., Kasaba, Y., Asozu, T., \& Reinisch, B. W. (2011). On the simultaneity of substorm onset between two hemispheres. Journal of Geophysical Research, 116(4), A04211. https://doi.org/10.1029/2010JA016174

Morioka, A., Miyoshi, Y., Tsuchiya, F., Misawa, H., Sakanoi, T., Yumoto, K., \& Donovan, E. F. (2007). Dual structure of auroral acceleration regions at substorm onsets as derived from auroral kilometric radiation spectra. Journal of Geophysical Research, 112(6), 1-13. https:// doi.org/10.1029/2006JA012186

Mutel, R. L., Christopher, I. W., \& Pickett, J. S. (2008). Cluster multispacecraft determination of AKR angular beaming. Geophysical Research Letters, 35(7), 1-6. https://doi.org/10.1029/2008GL033377

Mutel, R. L., Gumett, D. A., Christopher, I. W., Pickett, J. S., \& Schlax, M. (2003). Locations of auroral kilometric radiation bursts inferred from multispacecraft wideband Cluster VLBI observations. 1: Description of technique and initial results. Journal of Geophysical Research, 108(A11), 1-13. https://doi.org/10.1029/2003JA010011

Mutel, R. L., Gurnett, D. A., \& Christopher, I. W. (2004). Spatial and temporal properties of AKR burst emission derived from Cluster WBD VLBI studies. Annales Geophysicae, 22(7), 2625-2632. https://doi.org/10.5194/angeo-22-2625-2004

Novaco, J. C., \& Brown, L. W. (1978). Nonthermal galactic emission below 10 megahertz. The Astrophysical Journal, 221(A6), 114. https:// doi.org/10.1086/156009

Panchenko, M. (2003). Direction finding of AKR sources with three orthogonal antennas. Radio Science, 38(6), 1099. https://doi. org/10.1029/2003rs002929

Panchenko, M., Khodachenko, M. L., Kislyakov, A. G., Rucker, H. O., Hanasz, J., Kaiser, M. L., \& Goetz, K. (2009). Daily variations of auroral kilometric radiation observed by STEREO. Geophysical Research Letters, 36(6), 2-5. https://doi.org/10.1029/2008gl037042

Pulupa, M., Bale, S. D., Badman, S. T., Bonnell, J. W., Case, A. W., de Wit, T. D., \& Whittlesey, P. (2019). Statistics and polarization of type III radio bursts observed in the inner heliosphere. The Astrophysical Journal - Supplement Series, 246(2), 49. https://doi. org/10.3847/1538-4365/ab5dc0

Reiner, M. J., Fainberg, J., Kaiser, M. L., \& Bougeret, J. L. (2007). Circular polarization observed in interplanetary type III radio storms. Solar Physics, 241(2), 351-370. https://doi.org/10.1007/s11207-007-0277-8 
Schreiber, R., Panchenko, M., Hanasz, J., Mutel, R., \& Christopher, I. (2017). Beaming of intense AKR seen from the Interball-2 spacecraft. Journal of Geophysical Research: Space Physics, 122(1), 249-257. https://doi.org/10.1002/2015JA022197

Shue, J.-H., Song, P., Russell, C. T., Steinberg, J. T., Chao, J. K., Zastenker, G., \& Kawano, H. (1998). Magnetopause location under extreme solar wind conditions. Journal of Geophysical Research, 103(A8), 17691-17700. https://doi.org/10.1029/98ja01103

Voots, G. R., Gurnett, D. A., \& Akasofu, S. I. (1977). Auroral kilometric radiation as an indicator of auroral magnetic disturbances. Journal of Geophysical Research, 82(16), 2259-2266. https://doi.org/10.1029/ja082i016p02259

Wu, C. S., \& Lee, L. C. (1979). A theory of the terrestrial kilometric radiation. The Astrophysical Journal, 230, 621. https://doi. org/10.1086/157120

Wu, D. J., Chao, J. K., \& Lepping, R. P. (2000). Interaction between an interplanetary magnetic cloud and the Earth's magnetosphere: Motions of the bow shock. Journal of Geophysical Research, 105(A6), 12627-12638. https://doi.org/10.1029/1999ja000265

Zarka, P., Cecconi, B., \& Kurth, W. S. (2004). Jupiter's low-frequency radio spectrum from cassini/radio and plasma wave science (RPWS) absolute flux density measurements. Journal of Geophysical Research, 109(A9), 1-18. https://doi.org/10.1029/2003ja010260

Zaslavsky, A., Meyer-Vernet, N., Hoang, S., Maksimovic, M., \& Bale, S. D. (2011). On the antenna calibration of space radio instruments using the galactic background: General formulas and application to stereo/waves. Radio Science, 46(2), 1-7. https://doi. org/10.1029/2010RS004464

Zhao, W., Liu, S., Zhang, S., Zhou, Q., Yang, C., He, Y., \& Xiao, F. (2019). Global occurrences of auroral kilometric radiation related to suprathermal electrons in radiation belts. Geophysical Research Letters, 2, 7230-7236. https://doi.org/10.1029/2019GL083944 